\title{
A DECOUPLED ENERGY STABLE SCHEME FOR A HYDRODYNAMIC PHASE-FIELD MODEL OF MIXTURES OF NEMATIC LIQUID CRYSTALS AND VISCOUS FLUIDS
}

\author{
$\mathrm{JIA} \mathrm{ZHAO}^{\dagger}, \mathrm{XIAOFENG} \mathrm{YANG}^{\diamond}, \mathrm{JIE} \mathrm{SHEN}^{\triangleright}$ AND QI WANG $\ddagger^{\star}$
}

\begin{abstract}
We develop a linear, first-order, decoupled, energy-stable scheme for a two-phase hydrodynamic phase field model of mixtures of nematic liquid crystals and viscous fluids that satisfies an energy dissipation law. We show that the semi-discrete scheme in time satisfies an analogous, semi-discrete energy-dissipation law for any time-step and is therefore unconditional stable. We then discretize the spatial operators in the scheme by finite-differencing and implement the fully discrete scheme in a simplified version using CUDA on GPUs in 3 dimension in space. Two numerical examples for rupture of nematic liquid crystal filaments immersed in a viscous fluid matrix are given, illustrating the effectiveness of this new scheme in resolving complex interfacial phenomena in free surface flows of nematic liquid crystals.
\end{abstract}

\section{INTRODUCTION}

The two-phase phase field model, also known as the diffuse interface model, is a tool to resolve the motion of free interfaces between two fluid components, whose origin can be traced back to Rayleigh [23] and Van der Waals [29]. It was first formulated to study material mixtures and later adopted as a technique to resolve motion of the interface between different material components in material mixtures $[3,4,36]$. With the new development in advanced algorithms and computational technologies recently, it has emerged as an efficient numerical method to resolve complex dynamics in interfacial fluid flows involving complex topological changes. An advantage of the phase-field approach is that the model is often derived from a variational principle with an energy dissipation law, making it possible to carry out rigorous mathematical analyses, including the existence and uniqueness of the solution of the governing partial differential equations. It also makes it plausible to design the numerical scheme that obeys an analogous discrete energy dissipation law, warranting nonlinear stability in the numerical computation [1,11,12,21,22,34]. For more details about the numerical methods developed for phase field models, readers are referred to some recent review papers $[15,27]$ and the references therein.

Recently, Shen and Yang [28] developed a new numerical scheme for solving a hydrodynamic phase-field model for binary mixture flows of liquid crystals and viscous fluids, where the phase field transport equation is the Allen-Cahn equation [28]. Since the commonly used Allen-Cahn equation does not conserve the fluid volume, they used the Lagrangian multiplier method to enforce

Date: May 19, 2015.

$\dagger$ Department of Mathematics, University of South Carolina, Columbia, SC 29208; Email: zhao62@math.sc.edu.

$\diamond$ Department of Mathematics, University of South Carolina, Columbia, SC 29208; Email: xfyang@math.sc.edu.

$\triangle$ Department of Mathematics, Purdue University,West Lafayette, IN 47907; Email: shen@math.purdue.edu.

$\ddagger^{*}$ Corresponding author, Department of Mathematics, University of South Carolina, Columbia, SC 29208; Beijing Computational Science Research Center, Beijing, China, 100084; School of Mathematics, Nankai University, Tianjin, China, 300084; Email: qwang@math.sc.edu. 
the volume conservation property in their model. They proved that the new scheme satisfies a semi-discrete energy law, leading to decoupled, elliptic equations to solve at each time step, and is unconditionally stable in time. The type of schemes that obeys an analogous, discrete energy dissipation law is customarily called the energy stable scheme. In order to prove the energy stability for the scheme, however, they did not use the time invariant derivative in the director transport equation and in the meantime omitted the corresponding elastic stress for the liquid crystal component in the model. The mathematical convenience leads to a model that no longer describes the liquid crystal component well in the flow of the mixture fluid.

In this paper, we extend the previous study to a hydrodynamic phase field model for the binary mixture flow of liquid crystals and viscous fluids, in which the Cahn-Hilliard equation is adopted as the transport equation for the phase variable so that the volume of the mixture fluid is automatically conserved. We devise a new semi-discrete scheme in time and then prove the scheme is energy stable for the full model without any modification. This is by no means an easy task due to highly nonlinear couplings among the velocity, the hydrostatic pressure, the phase field function (or variable), the director field for liquid crystals, the time invariant derivative and the nontrivial elastic stress tensor. We note that this approach also applies to the Allen-Cahn model with the correct invariant time derivative for the director vector and elastic stress, extending the work of [28]. In addition, the anchoring energy, where liquid crystal has preferred orientation at the interface, has also been considered. This is, to our best knowleage, the first paper documented to tackle this issue. Specifically, we design an energy stable numerical scheme in a semi-discrete form in time and show that it satisfies the following properties: (a) it is unconditionally stable in time; (b) it satisfies a discrete energy law; and (c) it leads to decoupled, elliptic equations to solve at each time step so that fast solvers for elliptic equations can be applied. We then implement this numerical scheme on graphic processing units (GPUs) using CUDA to conduct the mesh refinement test and study rupture dynamics of a nematic liquid crystal drop or filament immersed in a viscous fluid [31]. Numerical examples demonstrate the desired accuracy in the time-step refinement test and capture some interesting phenomena when the liquid crystal filament ruptures.

The rest of the paper is organized as follows. In Section 2, we describe a hydrodynamic phasefield model for the mixture flow of nematic liquid crystals and viscous fluids using the CahnHilliard equation as the phase transport equation and derive the associated energy dissipation law. In Section 3, we develop the decoupled, energy stable numerical scheme for solving the coupled nonlinear hydrodynamic equation system. In section 4, we prove the semi-discrete energy law for the new numerical scheme. In Section 5, we present the mesh refinement results and a couple of numerical examples to illustrate the efficiency of the proposed scheme and study dynamics of nematic liquid crystal drops and filaments immersed in viscous fluid matrix.

\section{TWO-PHASE HYDRODYNAMIC MODEL FOR MIXTURES OF NEMATIC LIQUID CRYSTALS AND VISCOUS FLUIDS}

We consider a two-phase hydrodynamic phase field model for immiscible mixtures of nematic liquid crystals (LC) immersed in a viscous fluid matrix. We use a phase function $\phi$ to represent the volume fraction of the liquid crystal phase,

$$
\phi(x, t)= \begin{cases}1 & \text { liquid crystal } \\ 0 & \text { viscous fluid }\end{cases}
$$


with a thin smooth transitional layer of thickness $\varepsilon$ separating the liquid crystal from the viscous fluid. The interface of the mixture is described by the level set $\Gamma_{t}=\left\{x: \phi(x, t)=\frac{1}{2}\right\}$. We use $\rho$ and $\eta$ to denote the volume-averaged density and viscosity. Without loss of generality, we assume all model parameters are already non-dimensionalized and therefore dimensionless.

The total energy of the mixture fluid system is given by the sum of the kinetic energy $E_{k i n}$, the mixing free energy $E_{b}$, the bulk free energy for liquid crystals $E_{\mathbf{d}}$, and the anchoring energy for liquid crystals $E_{\text {anch }}[34]$ :

$$
E=E_{b}+E_{\mathbf{d}}+E_{\text {anch }}+E_{\text {kin }} .
$$

Specifically, we denote $f(\phi)=\frac{1}{\varepsilon^{2}} \phi^{2}(1-\phi)^{2}$ as the Ginzburg-Landau double-well potential and define the mixing free energy functional by

$$
E_{b}=\int_{\Omega} \gamma\left(\frac{1}{2}|\nabla \phi|^{2}+f(\phi)\right) d \mathbf{x}
$$

where $\gamma$ is the strength of the energy related to the traditional surface tension [34].

We assume that the bulk energy for liquid crystals is given by the modified Oseen-Frank distortional energy with a penalizing bulk term for handling potential defects $[8,16,17]$ :

$$
E_{\mathbf{d}}=\int_{\Omega} \frac{1}{2} \phi^{2} W(\mathbf{d}) d \mathbf{x}, \quad W(\mathbf{d})=K\left(\frac{1}{2}|\nabla \mathbf{d}|^{2}+g(\mathbf{d})\right)
$$

where $K$ is the Frank elastic constant [32] and $g(\mathbf{d})=\frac{1}{4 \delta^{2}}\left(|\mathbf{d}|^{2}-1\right)^{2}$ is a Ginzburg-Landau type penalty term, introduced to approximate the unit length constraint of $\mathbf{d}[18,19]$, where $\delta$ is a model parameter measuring the size of the defect core.

At the interface between the viscous fluid and the liquid crystal, a surface energy known as the anchoring energy is necessary to yield a preferred orientation for the liquid crystal $[6,13]$. The anchoring energy is given by

$$
E_{\text {anch }}=\int_{\Omega}\left[\frac{A_{1}}{2}(\mathbf{d} \cdot \nabla \phi)^{2}+\frac{A_{2}}{2}\left(|\mathbf{d}|^{2}|\nabla \phi|^{2}-(\mathbf{d} \cdot \nabla \phi)^{2}\right)\right] d \mathbf{x},
$$

where $A_{1}$ and $A_{2}\left(A_{1}>0, A_{2}>0\right)$ are the strength for the parallel and perpendicular anchoring energy, respectively.

The kinetic energy of the mixture system is

$$
E_{k i n}=\int_{\Omega} \frac{1}{2} \rho|\mathbf{u}|^{2} d \mathbf{x},
$$

where $\rho$ is the density of the mixture and $\mathbf{u}$ is the volume-averaged fluid velocity field.

Assuming (i). the phase field variable obeys the Cahn-Hilliard dynamics, (ii). the nematic director follows an Allen-Cahn dynamics [3, 9,20,21], (iii) the two fluids have a matching density $\rho=1$ and viscosity $\eta$, we obtain the following dimensionless governing system of equations:

$$
\left\{\begin{array}{l}
\partial_{t} \mathbf{u}+\mathbf{u} \cdot \nabla \mathbf{u}=-\nabla p+\eta \Delta \mathbf{u}+\nabla \cdot \tau_{e}-\phi \nabla \mu-\mathbf{h} \nabla \mathbf{d}, \\
\nabla \cdot \mathbf{u}=0, \\
\partial_{t} \mathbf{d}+\mathbf{u} \cdot \nabla \mathbf{d}-\mathbf{W} \cdot \mathbf{d}=a \mathbf{D} \cdot \mathbf{d}+M_{1} \mathbf{h}, \\
\partial_{t} \phi+\nabla \cdot(\mathbf{u} \phi)=M_{2} \Delta \mu,
\end{array}\right.
$$


where

$$
\begin{aligned}
\tau_{e} & =-\frac{a}{2}(\mathbf{d h}+\mathbf{h d})+\frac{1}{2}(\mathbf{d} \mathbf{h}-\mathbf{h d}), \\
\mu & =\gamma(\Delta \phi-f(\phi))-K \phi W(\mathbf{d})-\left(A_{1}-A_{2}\right) \nabla \cdot((\mathbf{d} \cdot \nabla \phi) \mathbf{d})-A_{2} \nabla \cdot\left(|\mathbf{d}|^{2} \nabla \phi\right), \\
\mathbf{h} & =\nabla \cdot\left(\frac{K \phi^{2}}{2} \nabla \mathbf{d}\right)+\frac{K \phi^{2}}{2} g^{\prime}(\mathbf{d})-\left(A_{1}-A_{2}\right)(\mathbf{d} \cdot \nabla \phi) \nabla \phi-A_{2}|\nabla \phi|^{2} \mathbf{d} .
\end{aligned}
$$

Here $\mu=\frac{\delta F}{\delta \phi}$ is the chemical potential [35], $\mathbf{h}=-\frac{\delta F}{\delta \mathbf{d}}$ the molecular field, $\tau_{e}$ is the elastic stress tensor associated with liquid crystal dynamics [32,33], $\mathbf{D}_{\alpha \beta}=\frac{1}{2}\left(\partial_{\beta} \mathbf{u}_{\alpha}+\partial_{\alpha} \mathbf{u}_{\beta}\right)$ is the rate of strain tensor, $\mathbf{W}_{\alpha \beta}=\frac{1}{2}\left(\partial_{\beta} \mathbf{u}_{\alpha}-\partial_{\alpha} \mathbf{u}_{\beta}\right)$ is the vorticity tensor, $p$ is the hydrostatic pressure, $1 / M_{1}$ is the relaxation time parameter of LC director dynamics, $M_{2}$ is the mobility parameter of the phase field function, a is a geometry parameter of liquid crystal molecules and $\eta$ is the volume-averaged viscosity.

Remark 2.1. When the two fluids have different densities with a relatively small density difference, one can use the Boussinesq approximation $[21,30]$. The case of different viscosities can usually be dealt with in a straightforward manner by assuming the viscosity is a linear or harmonic average of the phase function.

Throughout the paper, we assume the following boundary conditions

$$
\left.\mathbf{u}\right|_{\partial \Omega}=0,\left.\quad \nabla \phi \cdot \mathbf{n}\right|_{\partial \Omega}=0,\left.\quad \nabla \mu \cdot \mathbf{n}\right|_{\partial \Omega}=0,\left.\quad \nabla \mathbf{d} \cdot \mathbf{n}\right|_{\partial \Omega}=0
$$

with $\mathbf{n}$ the unit outward normal, which warrants the boundary effect will not contribute to the energy dissipation. In fact, all results presented in this paper are valid for periodic boundary conditions as well.

Notice the fact that this system is energy dissipative, which enables us to prove the existence and uniqueness of the weak solution with certain smoothness by a standard Galerkin procedure [7]. If we use $\mathscr{E}$ to denote the total energy density, its time rate of change is given by

$$
\begin{aligned}
\frac{d \mathscr{E}}{d t}= & \frac{\partial}{\partial t} \int_{\Omega}\left(\frac{1}{2} \mathbf{u}^{2}+E\right) d \mathbf{x} \\
= & \int_{\Omega} \mathbf{u} \cdot \partial_{t} \mathbf{u}+\frac{\delta E}{\delta \phi} \frac{\partial \phi}{\partial t}+\frac{\delta E}{\delta \mathbf{d}} \frac{\partial \mathbf{d}}{\partial t} d \mathbf{x} \\
= & \int_{\Omega} \mathbf{u} \cdot\left(-\mathbf{u} \cdot \nabla \mathbf{u}-\nabla p+\eta \Delta \mathbf{u}-\phi \nabla \mu-\mathbf{h} \nabla \mathbf{d}+\nabla \cdot\left(-\frac{a}{2}(\mathbf{d h}+\mathbf{h d})+\frac{1}{2}(\mathbf{d} \mathbf{h}-\mathbf{h d})\right)\right. \\
& +\mu\left(-\nabla \cdot(\mathbf{u} \phi)+M_{2} \Delta \mu\right)-\mathbf{h}\left(-\mathbf{u} \cdot \nabla \mathbf{d}+\mathbf{W} \cdot \mathbf{d}+a \mathbf{D} \cdot \mathbf{d}+M_{1} \mathbf{h}\right) d \mathbf{x} \\
= & \int_{\Omega}-\nabla \cdot\left(\mathbf{u} \frac{|\mathbf{u}|^{2}}{2}\right)+\frac{|\mathbf{u}|^{2}}{2} \nabla \cdot \mathbf{u}-\nabla \cdot(p \mathbf{u})+p \nabla \cdot \mathbf{u}-\nabla \cdot(\mu \phi \mathbf{u}) \\
& +\nabla \cdot\left(-\frac{a}{2}(\mathbf{d} \mathbf{h}+\mathbf{h d}) \mathbf{u}+\frac{1}{2}(\mathbf{d} \mathbf{h}-\mathbf{h} \mathbf{d}) \mathbf{u}\right)+\nabla \cdot(\eta \mathbf{u} \nabla \mathbf{u})-\eta|\nabla \mathbf{u}|^{2} \\
& -M_{1}|\mathbf{h}|^{2}+\nabla \cdot\left(M_{2} \mu \nabla \mu\right)-M_{2}|\nabla \mu|^{2} d \mathbf{x} \\
= & -\int_{\Omega}\left(\eta|\nabla \mathbf{u}|^{2}+M_{1}|\mathbf{h}|^{2}+M_{2}|\nabla \mu|^{2}\right) d \mathbf{x} .
\end{aligned}
$$

Clearly, the parameters $\eta, M_{1}$ and $M_{2}$ affect the magnitude of the dissipation rate. 


\section{Decoupled Semi-Discrete Scheme}

One of the desirable properties for the discretized dissipative system to have is to maintain its own energy dissipation law that is consistent with the energy law obeyed by the continuous differential system. Practically, this is an indication for a good approximation to the differential dissipative system. This type of numerical scheme is known as the energy stable scheme. In the following, we will design a semi-discrete energy stable scheme that addresses the following issues:

- the coupling of the velocity and pressure through the incompressible condition;

- the stiffness in the phase field equation and the director equation associated with the interfacial width $\varepsilon$ and the defect core size $\delta$;

- the nonlinear couplings among the momentum transport equation, the phase transport equation and the director equation.

In doing so, we develop an energy stable scheme based on a stabilization technique [25]. To prove energy stability of the scheme, we have to put some constraints on the potential function $f(\phi)$ and $g(\mathbf{d})$, i.e., they satisfy the following conditions: (i). $f$ and $g$ have continuous second order derivatives, (ii). there exist constants $L_{1}$ and $L_{2}$ such that

$$
\max _{|\mathbf{d}| \in \mathbb{R}^{3}}|H(\mathbf{d})| \leq L_{2}, \quad \max _{|\phi| \in \mathbb{R}}\left|f^{\prime \prime}(\phi)\right| \leq L_{1},
$$

where $H(\mathbf{d})$ is the Hessian matrix of $g(\mathbf{d})$.

One immediately notice that this condition is not satisfied by the usual double-well potentials $f(\phi)=\frac{1}{\varepsilon^{2}} \phi^{2}(\phi-1)^{2}$ and $g(\mathbf{d})=\frac{1}{4 \eta^{2}}\left(|\mathbf{d}|^{2}-1\right)^{2}$. However, we can modify $f(\phi)$ to quadratic growth outside of a physically meaningful interval $[-M, M]$ without affecting the solution if the maximum norm of the initial condition $\phi_{0}$ is bounded by $M$. Analogously, we can modify the function $g$ outside a ball in $\mathbb{R}^{3}$ of radius $M$. Therefore, it is common (cf. $[5,14,25]$ ) to consider the CahnHilliard equations with a modified double-well potential $\tilde{f}(\phi)$ and Allen-Cahn with a modified $\tilde{g}$. In the following, we drop the tilde $\tilde{\bullet}$ and assume both $f$ and $g$ satisfy the conditions (i) and (ii) listed above.

We now present the numerical scheme as follows.

\section{The semi-discrete scheme}

Given the initial conditions $\mathbf{d}^{0}, \phi^{0}, \mathbf{u}^{0}$ and $p^{0}=0$, having computed $\mathbf{d}^{n}, \phi^{n}, \mathbf{u}^{n}$ and $p^{n}$ for $n \geq 0$, we compute $\left(\mathbf{d}^{n+1}, \phi^{n+1}, \mathbf{u}^{n+1}, p^{n+1}\right)$ in the following sequence.

(1) Step 1: update $\mathbf{d}^{n+1}$ :

$$
\left\{\begin{aligned}
\dot{\mathbf{d}}^{n+1}= & M_{1} \mathbf{h}^{n+1} \\
\dot{\mathbf{d}}^{n+1}= & \frac{\mathbf{d}^{n+1}-\mathbf{d}^{n}}{\delta t}+\mathbf{u}_{\star}^{n} \cdot \nabla \mathbf{d}^{n}-\mathbf{W}_{\star}^{n} \cdot \mathbf{d}^{n}-a \mathbf{D}_{\star}^{n} \cdot \mathbf{d}^{n}, \\
\mathbf{h}^{n+1}= & -C_{1}^{n}\left(\mathbf{d}^{n+1}-\mathbf{d}^{n}\right)+\nabla \cdot\left(\frac{K}{2}\left(\phi^{n}\right)^{2} \nabla \mathbf{d}^{n+1}\right)-\frac{K}{2}\left(\phi^{n}\right)^{2} g^{\prime}\left(\mathbf{d}^{n}\right) \\
& -\left(A_{1}-A_{2}\right)\left(\mathbf{d}^{n} \cdot \nabla \phi^{n}\right) \nabla \phi^{n}-A_{2}\left|\nabla \phi^{n}\right|^{2} \mathbf{d}^{n+1}, \\
\left.\frac{\partial \mathbf{d}}{\partial \mathbf{n}}\right|_{\partial \Omega}= & 0,
\end{aligned}\right.
$$


with

$$
\begin{aligned}
& \mathbf{u}_{\star}^{n}=\mathbf{u}^{n}-\delta t \mathbf{h}^{n+1} \nabla \mathbf{d}^{n}+\delta t \nabla \cdot\left(\frac{1-a}{2} \mathbf{d}^{n} \mathbf{h}^{n+1}-\frac{1+a}{2} \mathbf{h}^{n+1} \mathbf{d}^{n}\right), \\
& \mathbf{W}_{\star}^{n}=\frac{1}{2}\left(\nabla \mathbf{u}_{\star}^{n}-\left(\nabla \mathbf{u}_{\star}^{n}\right)^{T}\right), \quad \mathbf{D}_{\star}^{n}=\frac{1}{2}\left(\nabla \mathbf{u}_{\star}^{n}+\left(\nabla \mathbf{u}_{\star}^{n}\right)^{T}\right) .
\end{aligned}
$$

We impose an additional boundary condition $\left.\mathbf{u}_{\star}^{n}\right|_{\partial \Omega}=\mathbf{0}$ in this step when physical boundary conditions are imposed instead of the periodic boundary condition. This condition sometimes is satisfied automatically if the liquid crystal phase (denoted by $\phi=1$ ) is completely suspended inside $\Omega$, namely, $\left.\phi\right|_{\partial \Omega}=0$ and $\left.\nabla \phi\right|_{\partial \Omega}=\mathbf{0}$. By definition, $\left.\mathbf{h}^{n+1}\right|_{\partial \Omega}=\mathbf{0}$ and $\left.\mathbf{u}_{\star}^{n}\right|_{\partial \Omega}=\left.\mathbf{u}_{n}\right|_{\partial \Omega}=\mathbf{0}$. Otherwise, this new boundary condition serves as a bona fide intermediate boundary condition for $\mathbf{d}^{n+1}$.

(2) Step 2: update $\phi^{n+1}$ :

$$
\left\{\begin{aligned}
\dot{\phi}^{n+1}= & M_{2} \Delta \mu^{n+1}, \\
\mu^{n+1}= & C_{2}^{n}\left(\phi^{n+1}-\phi^{n}\right)+C_{3}^{n}\left(\Delta \phi^{n+1}-\Delta \phi^{n}\right)+\gamma\left(-\Delta \phi^{n+1}+f^{\prime}\left(\phi^{n}\right)\right) \\
& +\phi^{n+1} W\left(\mathbf{d}^{n+1}\right)-\left(A_{1}-A_{2}\right) \nabla \cdot\left(\left(\mathbf{d}^{n} \cdot \nabla \phi^{n}\right) \mathbf{d}^{n+1}\right)-A_{2} \nabla \cdot\left(\left|\mathbf{d}^{n+1}\right|^{2} \nabla \phi^{n+1}\right), \\
\left.\frac{\partial \phi^{n+1}}{\partial \mathbf{n}}\right|_{\partial \Omega}= & 0,\left.\quad \frac{\partial \mu^{n+1}}{\partial \mathbf{n}}\right|_{\partial \Omega}=0,
\end{aligned}\right.
$$

with

$$
\begin{aligned}
& \dot{\phi}^{n+1}=\frac{\phi^{n+1}-\phi^{n}}{\delta t}+\nabla \cdot\left(\mathbf{u}_{\star \star}^{n} \phi^{n}\right), \\
& \mathbf{u}_{\star \star}^{n}=\mathbf{u}_{\star}^{n}-\delta t \phi^{n} \nabla \mu^{n+1}, \\
& W\left(\mathbf{d}^{n+1}\right)=K\left(\frac{1}{2}\left|\nabla \mathbf{d}^{n+1}\right|^{2}+g\left(\mathbf{d}^{n+1}\right)\right) .
\end{aligned}
$$

(3) Step 3: update $\mathbf{u}^{n+1}$ :

$$
\left\{\begin{aligned}
\frac{\tilde{\mathbf{u}}^{n+1}-\mathbf{u}^{n}}{\delta t}+ & \left(\mathbf{u}^{n} \cdot \nabla\right) \tilde{\mathbf{u}}^{n+1}=\eta \Delta \tilde{\mathbf{u}}^{n+1}-\nabla p^{n}-\phi^{n} \nabla \mu^{n+1} \\
& -\mathbf{h}^{n+1} \nabla \mathbf{d}^{n}+\nabla \cdot\left(-\frac{a}{2}\left(\mathbf{d}^{n} \mathbf{h}^{n+1}+\mathbf{h}^{n+1} \mathbf{d}^{n}\right)+\frac{1}{2}\left(\mathbf{d}^{n} \mathbf{h}^{n+1}-\mathbf{h}^{n+1} \mathbf{d}^{n}\right)\right), \\
\left.\tilde{\mathbf{u}}^{n+1}\right|_{\partial \Omega}= & 0 .
\end{aligned}\right.
$$

$$
\left\{\begin{aligned}
\frac{\mathbf{u}^{n+1}-\tilde{\mathbf{u}}^{n+1}}{\delta t} & =-\nabla\left(p^{n+1}-p^{n}\right), \\
\nabla \cdot \mathbf{u}^{n+1} & =0,\left.\quad \mathbf{u}^{n+1}\right|_{\partial \Omega}=0 .
\end{aligned}\right.
$$

In the above, $C_{1}^{n}, C_{2}^{n}$ and $C_{3}^{n}$ are stabilizing parameters to be determined. The above scheme is constructed by combining several effective approaches in the approximation of Cahn-Hilliard equation [25], Navier-Stokes equations [10] and phase-field models [2,26].

Remark 3.1. A pressure-correction scheme [10] is used to decouple the computation of the pressure from that of the velocity.

Remark 3.2. We note that the explicit discretization of $f^{\prime}(\phi)=\frac{2}{\varepsilon^{2}} \phi(1-\phi)(1-2 \phi)$ often leads to a severe restriction on the time step $\delta t$ when $\varepsilon \ll 1$. Thus, we introduce a "stabilizing" term to improve stability while preserving simplicity in (5.5), which allows us to treat the nonlinear term explicitly without subject to any time step constraint [24-26]. This stabilizing term introduces an 
error of order $O(\delta t)$ in a small region near the interface, the same order as the error introduced by treating $f(\phi)$ explicitly; so the overall truncation error of the scheme is essentially the same with or without the stabilizing term. A similar approach is applied to the director equation for the treatment of $g(\mathbf{d})$.

Remark 3.3. The scheme given by (3.2)-(5.8) is a fully decoupled, linear scheme. Hence, one only needs to solve a series of elliptic equations, which can be done very efficiently using fast solvers. Of course, some of these elliptic equations may be of variable coefficients.

Remark 3.4. If we don't study the embedded phase and boundary interaction, the new intermediate boundary condition on $\mathbf{d}^{n+1}$ in practice can be avoided so long as we don't allow the embedded phase denoted by $\phi=1$ to touch the boundary. This condition is unnecessary if we deal with a periodic boundary condition.

We shall show next that the above scheme is energy stable unconditionally assuming each step can be solved uniquely.

\section{Semi-Discrete Energy Dissipation LaW}

In this section, we prove that the scheme derived in the previous section is unconditionally energy stable. Instead of going directly to the proof, we first provide some lemmas to help readers to better follow the details of the proof.

Lemma 4.1. Denote $\delta \phi^{n+1}=\phi^{n+1}-\phi^{n}$ and $\delta \delta \phi^{n+1}=\phi^{n+1}-2 \phi^{n}+\phi^{n-1}$. Then, the following equalities hold,

$$
\begin{array}{ll}
2\left(\phi^{n+1}-\phi^{n}, \phi^{n+1}\right) & =\left\|\phi^{n+1}\right\|^{2}-\left\|\phi^{n}\right\|^{2}+\left\|\phi^{n+1}-\phi^{n}\right\|^{2}, \\
2\left(\phi^{n+1}-\phi^{n}, \phi^{n}\right) & =\left\|\phi^{n+1}\right\|^{2}-\left\|\phi^{n}\right\|^{2}-\left\|\phi^{n+1}-\phi^{n}\right\|^{2}, \\
2\left(\nabla \phi^{n+1}-\nabla \phi^{n}, \nabla \phi^{n+1}\right) & +\left\|\nabla \phi^{n+1}\right\|^{2}-\left\|\nabla \phi^{n}\right\|^{2}+\left\|\nabla \phi^{n+1}-\nabla \phi^{n}\right\|^{2}, \\
2\left(\nabla \phi^{n+1}-\nabla \phi^{n}, \nabla \phi^{n}\right) & =\left\|\nabla \phi^{n+1}\right\|^{2}-\left\|\nabla \phi^{n}\right\|^{2}-\left\|\nabla \phi^{n+1}-\nabla \phi^{n}\right\|^{2} .
\end{array}
$$

Proof. This is trivial. We can obtain the equalities by simply expanding the inner product on the right hand side and then combining the common terms.

Lemma 4.2. If $F \in C^{2}\left(\mathbb{R}^{k}\right)$, where $k$ is the dimension, and

$$
\max _{\mathbf{x} \in \mathbb{R}^{k}}\left|F^{\prime \prime}(\mathbf{x})\right|<L,
$$

$\forall \mathbf{x}^{n+1}, \mathbf{x}^{n} \in \mathbb{R}^{k}$, the following inequality holds,

$$
\left(\mathbf{x}^{n+1}-\mathbf{x}^{n}, F^{\prime}\left(\mathbf{x}^{n}\right)\right) \geq\left(F\left(\mathbf{x}^{n+1}\right)-F\left(\mathbf{x}^{n}\right), 1\right)-L\left\|\mathbf{x}^{n+1}-\mathbf{x}^{n}\right\|^{2} .
$$

Proof. Notice the fact,

$$
\begin{aligned}
\left(F\left(\mathbf{x}^{n+1}\right)-F\left(\mathbf{x}^{n}\right), 1\right) & =\left(\int_{\mathbf{x}^{n}}^{\mathbf{x}^{n+1}} F^{\prime}(\mathbf{x}) d \mathbf{x}, 1\right) \\
& =\left(\left(\mathbf{x}^{n+1}-\mathbf{x}^{n}\right) F^{\prime}\left(\mathbf{x}^{n}\right)+\int_{\mathbf{x}^{n}}^{\mathbf{x}^{n+1}}\left(\mathbf{x}^{n+1}-\mathbf{x}\right) F^{\prime \prime}(\mathbf{x}) d \mathbf{x}, 1\right) \\
& \leq\left(\mathbf{x}^{n+1}-\mathbf{x}^{n}, F^{\prime}\left(\mathbf{x}^{n}\right)\right)+L\left\|\mathbf{x}^{n+1}-\mathbf{x}^{n}\right\|^{2} .
\end{aligned}
$$

Then, we obtain

$$
\left(\mathbf{x}^{n+1}-\mathbf{x}^{n}, F^{\prime}\left(\mathbf{x}^{n}\right)\right) \geq\left(F\left(\mathbf{x}^{n+1}\right)-F\left(\mathbf{x}^{n}\right), 1\right)-L\left\|\mathbf{x}^{n+1}-\mathbf{x}^{n}\right\|^{2} .
$$


Lemma 4.3. The following identity holds,

$$
\int_{\Omega}(\mathbf{v} \cdot \nabla) \mathbf{u} \cdot \mathbf{u} d \mathbf{x}=0
$$

provided that $\left.\mathbf{n} \cdot \mathbf{v}\right|_{\Gamma}=0, \mathbf{v}$ and $\mathbf{u}$ are sufficiently smooth and $\nabla \cdot \mathbf{v}=0$, where $\mathbf{n}$ is the unit external normal of the surface.

Proof. It is straightforward to show the following:

$$
\begin{aligned}
\int_{\Omega}(\mathbf{v} \cdot \nabla) \mathbf{u} \cdot \mathbf{u} d \mathbf{x} & =\int_{\Omega} \nabla \cdot\left(\mathbf{v} \frac{|\mathbf{u}|^{2}}{2}\right)-\frac{|\mathbf{u}|^{2}}{2} \nabla \cdot \mathbf{v} d \mathbf{x} \\
& =\int_{\partial \Omega} \mathbf{n} \cdot \mathbf{v} \frac{|\mathbf{u}|^{2}}{2} d s \\
& =0
\end{aligned}
$$

Lemma 4.4. If $C_{1}^{n}, C_{2}^{n}$ and $C_{3}^{n}$ satisfy the following conditions,

$$
\begin{aligned}
& C_{1}^{n} \geq \frac{K L_{2}}{2}\left\|\phi^{n}\right\|_{\infty}^{2}+\frac{1}{2} \max \left(A_{1}-2 A_{2}, 0\right)\left\|\nabla \phi^{n}\right\|_{\infty}, \\
& C_{2}^{n} \geq \gamma L_{1}, \\
& C_{3}^{n} \geq \frac{1}{2} \max \left(A_{1}-2 A_{2}, 0\right)\left\|\mathbf{d}^{n+1}\right\|_{\infty}-\frac{\gamma}{2},
\end{aligned}
$$

then

$$
\left(\phi^{n+1}-\phi^{n}, \mu^{n+1}\right)-\left(\mathbf{d}^{n+1}-\mathbf{d}^{n}, \mathbf{h}^{n+1}\right) \geq F^{n+1}-F^{n},
$$

where $F^{n}$ is the semi-discrete free energy defined as

$$
\begin{aligned}
& F^{n}=F_{b}^{n}+F_{\mathbf{d}}^{n}+F_{\text {anch }}^{n}, \\
& \left.F_{b}^{n}=\gamma\left(\frac{1}{2}\left|\nabla \phi^{n}\right|^{2}+f\left(\phi^{n}\right), 1\right)\right), \\
& F_{\mathbf{d}}^{n}=\left(\frac{1}{2}\left(\phi^{n}\right)^{2} W\left(\mathbf{d}^{n}\right), 1\right), \quad W\left(\mathbf{d}^{n}\right)=K\left(\frac{1}{2}\left|\nabla \mathbf{d}^{n}\right|^{2}+g\left(\mathbf{d}^{n}\right)\right), \\
& F_{\text {anch }}^{n}=\frac{A_{1}}{2}\left(\left(\nabla \phi^{n} \cdot \mathbf{d}^{n}\right)^{2}, 1\right)+\frac{A_{2}}{2}\left(\left|\mathbf{d}^{n}\right|^{2}\left|\nabla \phi^{n}\right|^{2}-\left(\nabla \phi^{n} \cdot \mathbf{d}^{n}\right)^{2}, 1\right) .
\end{aligned}
$$

Proof. Using the definition of $\mathbf{u}^{n+1}$ in (5.5) and $\mathbf{h}^{n+1}$ in (3.2), the first term on the left hand in (4.9) can be expanded as

$$
\begin{aligned}
& \left(\phi^{n+1}-\phi^{n}, \mu^{n+1}\right)=C_{2}^{n}\left\|\phi^{n+1}-\phi^{n}\right\|^{2}+C_{3}^{n}\left\|\nabla \phi^{n+1}-\nabla \phi^{n}\right\|^{2} \\
& +\frac{\gamma}{2}\left(\left\|\nabla \phi^{n+1}\right\|^{2}-\left\|\nabla \phi^{n}\right\|^{2}+\left\|\nabla \phi^{n+1}-\nabla \phi^{n}\right\|^{2}\right)+\gamma\left(\phi^{n+1}-\phi^{n}, f^{\prime}\left(\phi^{n}\right)\right) \\
& +\left(\phi^{n+1}-\phi^{n}, \phi^{n+1} W\left(\mathbf{d}^{n+1}\right)\right)-\left(\phi^{n+1}-\phi^{n},\left(A_{1}-A_{2}\right) \nabla \cdot\left(\left(\mathbf{d}^{n} \cdot \nabla \phi^{n}\right) \mathbf{d}^{n+1}\right)\right. \\
& +\left(\phi^{n+1}-\phi^{n}, A_{2} \nabla \cdot\left(\left|\mathbf{d}^{n+1}\right|^{2} \nabla \phi^{n+1}\right)\right) .
\end{aligned}
$$


The second term on the left hand in (4.9) can be rewritten into the following

$$
\begin{aligned}
& -\left(\mathbf{d}^{n+1}-\mathbf{d}^{n}, \mathbf{h}^{n+1}\right)=C_{1}^{n}\left\|\mathbf{d}^{n+1}-\mathbf{d}^{n}\right\|^{2}-\left(\mathbf{d}^{n+1}-\mathbf{d}^{n}, \nabla \cdot\left(\frac{K}{2}\left(\phi^{n}\right)^{2} \nabla \mathbf{d}^{n+1}\right)\right) \\
& +\left(\mathbf{d}^{n+1}-\mathbf{d}^{n}, \frac{K}{2}\left(\phi^{n}\right)^{2} g^{\prime}\left(\mathbf{d}^{n}\right)\right)+\left(A_{1}-A_{2}\right)\left(\mathbf{d}^{n+1}-\mathbf{d}^{n},\left(\mathbf{d}^{n} \cdot \nabla \phi^{n}\right) \nabla \phi^{n}\right) \\
& +A_{2}\left(\mathbf{d}^{n+1}-\mathbf{d}^{n},\left|\nabla \phi^{n}\right|^{2} \mathbf{d}^{n+1}\right) .
\end{aligned}
$$

We denote

$$
\begin{aligned}
T & =\left(\phi^{n+1}-\phi^{n}, \mu^{n+1}\right) \\
T_{0} & =C_{2}^{n}\left\|\phi^{n+1}-\phi^{n}\right\|^{2}+C_{3}^{n}\left\|\nabla \phi^{n+1}-\nabla \phi^{n}\right\|^{2}, \\
T_{1} & =\gamma\left(\phi^{n+1}-\phi^{n}, f^{\prime}\left(\phi^{n}\right)\right)+\frac{\gamma}{2}\left(\left\|\nabla \phi^{n+1}\right\|^{2}-\left\|\nabla \phi^{n}\right\|^{2}+\left\|\nabla \phi^{n+1}-\nabla \phi^{n}\right\|^{2}\right), \\
T_{2} & =\left(\phi^{n+1}-\phi^{n}, \phi^{n+1} W\left(\mathbf{d}^{n+1}\right)\right), \\
T_{3} & =-\left(A_{1}-A_{2}\right)\left(\phi^{n+1}-\phi^{n}, \nabla \cdot\left(\left(\mathbf{d}^{n} \cdot \nabla \phi^{n}\right) \mathbf{d}^{n+1}\right)\right), \\
T_{4} & =-A_{2}\left(\phi^{n+1}-\phi^{n}, \nabla \cdot\left(\left|\mathbf{d}^{n+1}\right|^{2} \nabla \phi^{n+1}\right)\right) .
\end{aligned}
$$

In addition, we introduce

$$
\begin{aligned}
P & =-\left(\mathbf{d}^{n+1}-\mathbf{d}^{n}, \mathbf{h}^{n+1}\right), \\
P_{0} & =C_{1}^{n}\left\|\mathbf{d}^{n+1}-\mathbf{d}^{n}\right\|^{2}, \\
P_{1} & =-\left(\mathbf{d}^{n+1}-\mathbf{d}^{n}, \nabla \cdot\left(\frac{K}{2}\left(\phi^{n}\right)^{2} \nabla \mathbf{d}^{n+1}\right)\right), \\
P_{2} & =\left(\mathbf{d}^{n+1}-\mathbf{d}^{n}, \frac{K}{2}\left(\phi^{n}\right)^{2} g^{\prime}\left(\mathbf{d}^{n}\right)\right), \\
P_{3} & =\left(A_{1}-A_{2}\right)\left(\mathbf{d}^{n+1}-\mathbf{d}^{n},\left(\mathbf{d}^{n} \cdot \nabla \phi^{n}\right) \nabla \phi^{n}\right), \\
P_{4} & =A_{2}\left(\mathbf{d}^{n+1}-\mathbf{d}^{n},\left|\nabla \phi^{n}\right|^{2} \mathbf{d}^{n+1}\right),
\end{aligned}
$$

such that

$$
T=\sum_{i=0}^{4} T_{i}, \quad P=\sum_{i=0}^{4} P_{i} .
$$

Next, we analyze these terms one-by-one. For $T_{1}$, we have,

$$
\begin{aligned}
T_{1} & =\gamma\left(\phi^{n+1}-\phi^{n}, f^{\prime}\left(\phi^{n}\right)\right)+\frac{\gamma}{2}\left(\left\|\nabla \phi^{n+1}\right\|^{2}-\left\|\nabla \phi^{n}\right\|^{2}+\left\|\nabla \phi^{n+1}-\nabla \phi^{n}\right\|^{2}\right) \\
& =\gamma\left(f\left(\phi^{n+1}\right)-f\left(\phi^{n}\right), 1\right)-\gamma L_{1}\left\|\phi^{n+1}-\phi^{n}\right\|^{2}+\frac{\gamma}{2}\left(\left\|\nabla \phi^{n+1}\right\|^{2}-\left\|\nabla \phi^{n}\right\|^{2}+\left\|\nabla \phi^{n+1}-\nabla \phi^{n}\right\|^{2}\right),
\end{aligned}
$$

i.e.

$$
T_{1} \geq F_{b}^{n+1}-F_{b}^{n}+\frac{\gamma}{2}\left\|\nabla \phi^{n+1}-\nabla \phi^{n}\right\|^{2}-\gamma L_{1}\left\|\phi^{n+1}-\phi^{n}\right\|^{2}
$$


For $T_{2}$, we have,

$$
\begin{aligned}
T_{2} & =\left(\phi^{n+1}-\phi^{n}, \phi^{n+1} W\left(\mathbf{d}^{n+1}\right)\right) \\
& =\left(\frac{1}{2}\left(\phi^{n+1}\right)^{2}-\frac{1}{2}\left(\phi^{n}\right)^{2}, W\left(\mathbf{d}^{n+1}\right)\right)+\frac{1}{2}\left(\left(\phi^{n+1}-\phi^{n}\right)^{2}, W\left(\mathbf{d}^{n+1}\right)\right) \\
& \geq\left(\frac{1}{2}\left(\phi^{n+1}\right)^{2}-\frac{1}{2}\left(\phi^{n}\right)^{2}, W\left(\mathbf{d}^{n+1}\right)\right),
\end{aligned}
$$

if $W(\mathbf{d}) \geq 0, \forall \mathbf{d} \in \mathbb{R}^{3}$, which is true in our case.

For $P_{1}$, we have

$$
\begin{aligned}
P_{1} & =\left(\nabla \mathbf{d}^{n+1}-\nabla \mathbf{d}^{n}, \frac{K}{2}\left(\phi^{n}\right)^{2} \nabla \mathbf{d}^{n+1}\right) \\
& =\left(\frac{K}{2}\left(\phi^{n}\right)^{2}, \frac{1}{2}\left(\nabla \mathbf{d}^{n+1}\right)^{2}-\frac{1}{2}\left(\nabla \mathbf{d}^{n}\right)^{2}\right)+\left(\frac{K}{2}\left(\phi^{n}\right)^{2},\left(\nabla \mathbf{d}^{n+1}-\nabla \mathbf{d}^{n}\right)^{2}\right) \\
& \geq\left(\frac{K}{2}\left(\phi^{n}\right)^{2}, \frac{1}{2}\left(\nabla \mathbf{d}^{n+1}\right)^{2}-\frac{1}{2}\left(\nabla \mathbf{d}^{n}\right)^{2}\right) .
\end{aligned}
$$

For $P_{2}$, we use Lemma 4.2 and obtain

$$
\begin{aligned}
P_{2} & \geq\left(\frac{K}{2}\left(\phi^{n}\right)^{2}, g\left(\mathbf{d}^{n+1}\right)-g\left(\mathbf{d}^{n}\right)\right)-\left(\frac{K}{2}\left(\phi^{n}\right)^{2}, L_{2}\left(\mathbf{d}^{n+1}-\mathbf{d}^{n}\right)^{2}\right) \\
& \geq\left(\frac{K}{2}\left(\phi^{n}\right)^{2}, g\left(\mathbf{d}^{n+1}\right)-g\left(\mathbf{d}^{n}\right)\right)-\frac{L_{2} K}{2}\left\|\left(\phi^{n}\right)^{2}\right\|_{\infty}\left\|\mathbf{d}^{n+1}-\mathbf{d}^{n}\right\|^{2} .
\end{aligned}
$$

Combining $T_{2}, P_{1}$ and $P_{2}$, we obtain

$$
T_{2}+P_{1}+P_{2} \geq F_{\mathbf{d}}^{n+1}-F_{\mathbf{d}}^{n}-\frac{L_{2} K}{2}\left\|\left(\phi^{n}\right)^{2}\right\|_{\infty}\left\|\mathbf{d}^{n+1}-\mathbf{d}^{n}\right\|^{2} .
$$

Combining $T_{3}$ and $P_{3}$, we have

$$
\begin{aligned}
& T_{3}+P_{3} \\
= & \left(A_{1}-A_{2}\right)\left(\left(\mathbf{d}^{n+1}-\mathbf{d}^{n},\left(\mathbf{d}^{n} \cdot \nabla \phi^{n}\right) \nabla \phi^{n}\right)-\left(\phi^{n+1}-\phi^{n}, \nabla \cdot\left(\left(\mathbf{d}^{n} \cdot \nabla \phi^{n}\right) \mathbf{d}^{n+1}\right)\right)\right) \\
= & \left(A_{1}-A_{2}\right)\left(\left(\mathbf{d}^{n+1}-\mathbf{d}^{n},\left(\mathbf{d}^{n} \cdot \nabla \phi^{n}\right) \nabla \phi^{n}\right)+\left(\nabla \phi^{n+1}-\nabla \phi^{n},\left(\mathbf{d}^{n} \cdot \nabla \phi^{n}\right) \mathbf{d}^{n+1}\right)\right) \\
= & \left(A_{1}-A_{2}\right)\left(\left(\mathbf{d}^{n} \cdot \nabla \phi^{n}, \mathbf{d}^{n+1} \cdot \nabla \phi^{n+1}\right)-\left(\mathbf{d}^{n} \cdot \nabla \phi^{n}, \mathbf{d}^{n} \cdot \nabla \phi^{n}\right)\right) \\
= & \frac{A_{1}-A_{2}}{2}\left(\left\|\mathbf{d}^{n+1} \cdot \nabla \phi^{n+1}\right\|^{2}-\left\|\mathbf{d}^{n} \cdot \nabla \phi^{n}\right\|^{2}-\left\|\mathbf{d}^{n+1} \cdot \nabla \phi^{n+1}-\mathbf{d}^{n} \cdot \nabla \phi^{n}\right\|^{2}\right) .
\end{aligned}
$$


Adding $T_{4}$ with $P_{4}$, we have

$$
\begin{aligned}
& T_{4}+P_{4} \\
= & A_{2}\left(\left(\nabla \phi^{n+1}-\nabla \phi^{n},\left|\mathbf{d}^{n+1}\right|^{2} \nabla \phi^{n+1}\right)+\left(\mathbf{d}^{n+1}-\mathbf{d}^{n},\left|\nabla \phi^{n}\right|^{2} \mathbf{d}^{n+1}\right)\right) \\
= & \frac{A_{2}}{2}\left(\left(\left|\nabla \phi^{n+1}\right|^{2}-|\nabla \phi|^{2}+\left|\nabla \phi^{n+1}-\nabla \phi^{n}\right|^{2},\left|\mathbf{d}^{n+1}\right|^{2}\right)+\right. \\
& \left.\left(\left|\mathbf{d}^{n+1}\right|^{2}-\left|\mathbf{d}^{n}\right|^{2}+\left|\mathbf{d}^{n+1}-\mathbf{d}^{n}\right|^{2},\left|\nabla \phi^{n}\right|^{2}\right)\right) \\
= & \frac{A_{2}}{2}\left(\left|\mathbf{d}^{n+1}\right|^{2}\left|\nabla \phi^{n+1}\right|^{2}-\left|\mathbf{d}^{n}\right|^{2}\left|\nabla \phi^{n}\right|^{2}, 1\right)+\frac{A_{2}}{2}\left(\left|\nabla \phi^{n+1}-\nabla \phi^{n}\right|^{2},\left|\mathbf{d}^{n+1}\right|^{2}\right)+ \\
& \frac{A_{2}}{2}\left(\left|\mathbf{d}^{n+1}-\mathbf{d}^{n}\right|^{2},\left|\nabla \phi^{n}\right|^{2}\right) .
\end{aligned}
$$

Combining $T_{3}, T_{4}, P_{3}$ and $P_{4}$, we have

$$
\begin{aligned}
T_{3}+T_{4}+P_{3}+P_{4}= & F_{\text {anch }}^{n+1}-F_{\text {anch }}^{n}-\frac{A_{1}-A_{2}}{2}\left(\left\|\mathbf{d}^{n+1} \cdot \nabla \phi^{n+1}-\mathbf{d}^{n} \cdot \nabla \phi^{n}\right\|^{2}\right) \\
& +\frac{A_{2}}{2}\left(\left|\nabla \phi^{n+1}-\nabla \phi^{n}\right|^{2},\left|\mathbf{d}^{n+1}\right|^{2}\right)+\frac{A_{2}}{2}\left(\left|\mathbf{d}^{n+1}-\mathbf{d}^{n}\right|^{2},\left|\nabla \phi^{n}\right|^{2}\right) .
\end{aligned}
$$

Note that

$$
\begin{aligned}
& -\left\|\mathbf{d}^{n+1} \cdot \nabla \phi^{n+1}-\mathbf{d}^{n} \cdot \nabla \phi^{n}\right\|^{2} \\
= & -\left\|\mathbf{d}^{n+1} \cdot \nabla \phi^{n+1}-\mathbf{d}^{n+1} \cdot \nabla \phi^{n}+\mathbf{d}^{n+1} \cdot \nabla \phi^{n}-\mathbf{d}^{n} \cdot \nabla \phi^{n}\right\|^{2} \\
\geq & -\left\|\mathbf{d}^{n+1} \cdot\left(\nabla \phi^{n+1}-\nabla \phi^{n}\right)\right\|^{2}-\left\|\nabla \phi^{n} \cdot\left(\mathbf{d}^{n+1}-\mathbf{d}^{n}\right)\right\|^{2} \\
\geq & -\left(\left|\mathbf{d}^{n+1}\right|^{2},\left|\nabla \phi^{n+1}-\nabla \phi^{n}\right|^{2}\right)-\left(\left|\nabla \phi^{n}\right|^{2},\left|\mathbf{d}^{n+1}-\mathbf{d}^{n}\right|^{2}\right) .
\end{aligned}
$$

Then, we have

$$
\begin{aligned}
& T_{3}+T_{4}+P_{3}+P_{4} \geq F_{\text {anch }}^{n+1}-F_{\text {anch }}^{n} \\
& -\frac{1}{2} \max \left(A_{1}-2 A_{2}, 0\right)\left(\left\|\left(\mathbf{d}^{n+1}\right)^{2}\right\|_{\infty}\left\|\nabla \phi^{n+1}-\nabla \phi^{n}\right\|^{2}+\left\|\left(\nabla \phi^{n}\right)^{2}\right\|_{\infty}\left\|\mathbf{d}^{n+1}-\mathbf{d}^{n}\right\|^{2}\right) .
\end{aligned}
$$


Adding up (4.17),(4.21), (4.26), $T_{0}$ and $P_{0}$, we have

$$
\begin{aligned}
& \left(\phi^{n+1}-\phi^{n}, \mu^{n+1}\right)-\left(\mathbf{d}^{n+1}-\mathbf{d}^{n}, \mathbf{h}^{n+1}\right) \\
= & \sum_{i=0}^{4} T_{i}+\sum_{i=0}^{4} P_{i} \\
\geq & F^{n+1}-F^{n}+C_{2}^{n}\left\|\phi^{n+1}-\phi^{n}\right\|^{2}+C_{3}^{n}\left\|\nabla \phi^{n+1}-\nabla \phi^{n}\right\|^{2}+C_{1}^{n}\left\|\mathbf{d}^{n+1}-\mathbf{d}^{n}\right\|^{2} \\
& +\frac{\gamma}{2}\left\|\nabla \phi^{n+1}-\nabla \phi^{n}\right\|^{2}-\gamma L_{1}\left\|\phi^{n+1}-\phi^{n}\right\|^{2}-\frac{K L_{2}}{2}\left\|\phi^{n}\right\|_{\infty}^{2}\left\|\mathbf{d}^{n+1}-\mathbf{d}^{n}\right\|^{2} \\
& -\frac{1}{2} \max \left(A_{1}-2 A_{2}, 0\right)\left(\left\|\left(\mathbf{d}^{n+1}\right)^{2}\right\|_{\infty}\left\|\nabla \phi^{n+1}-\nabla \phi^{n}\right\|^{2}+\left\|\left(\nabla \phi^{n}\right)^{2}\right\|_{\infty}\left\|\mathbf{d}^{n+1}-\mathbf{d}^{n}\right\|^{2}\right) \\
= & F^{n+1}-F^{n}+\left(C_{1}^{n}-\frac{K L_{2}}{2}\left\|\phi^{n}\right\|_{\infty}^{2}--\frac{1}{2} \max \left(A_{1}-2 A_{2}, 0\right)\left\|\nabla \phi^{n}\right\|_{\infty}\right)\left\|\mathbf{d}^{n+1}-\mathbf{d}^{n}\right\|^{2} \\
& +\left(C_{2}^{n}-\gamma L_{1}\right)\left\|\nabla \phi^{n+1}-\nabla \phi^{n}\right\|^{2}+\left(C_{3}^{n}+\frac{\gamma}{2}-\frac{1}{2} \max \left(A_{1}-2 A_{2}, 0\right)\left\|\mathbf{d}^{n+1}\right\|_{\infty}\right)\left\|\nabla \phi^{n+1}-\nabla \phi^{n}\right\|^{2} .
\end{aligned}
$$

By the assumption, we have,

Finally, we arrive at

$$
\begin{aligned}
C_{1}^{n} & \geq \frac{K L_{2}}{2}\left\|\phi^{n}\right\|_{\infty}^{2}+\frac{1}{2} \max \left(A_{1}-2 A_{2}, 0\right)\left\|\nabla \phi^{n}\right\|_{\infty}, \\
C_{2}^{n} & \geq \gamma L_{1}, \\
C_{3}^{n} & \geq \frac{1}{2} \max \left(A_{1}-2 A_{2}, 0\right)\left\|\mathbf{d}^{n+1}\right\|_{\infty}-\frac{\gamma}{2} .
\end{aligned}
$$

$$
\left(\phi^{n+1}-\phi^{n}, \mu^{n+1}\right)-\left(\mathbf{d}^{n+1}-\mathbf{d}^{n}, \mathbf{h}^{n+1}\right) \geq F^{n+1}-F^{n} .
$$

Theorem 4.1. Under the conditions given in Lemma 4.4, the scheme given by (3.2)-(5.8) admits a unique solution satisfying the following semi-discrete energy dissipation law:

$$
\begin{aligned}
& \frac{1}{2}\left\|\mathbf{u}^{n+1}\right\|^{2}+F^{n+1}+\frac{\delta t^{2}}{2}\left\|\nabla p^{n+1}\right\|^{2}+\delta t\left(\eta\left\|\nabla \tilde{\mathbf{u}}^{n+1}\right\|^{2}+M_{2}\left\|\nabla \mu^{n+1}\right\|^{2}+M_{1}\left\|\mathbf{h}^{n+1}\right\|^{2}\right) \\
& \leq \frac{1}{2}\left\|\mathbf{u}^{n}\right\|^{2}+F^{n}+\frac{\delta t^{2}}{2}\left\|\nabla p^{n}\right\|^{2},
\end{aligned}
$$

where the semi-discrete energy $F^{n}$ is defined in (4.10).

Proof. From the definition of $\mathbf{u}_{\star}^{n}$ in equation (5.4) and $\mathbf{u}_{\star \star}^{n}$ in equation (5.6), we can rewrite the momentum equation as follows

$$
\frac{\tilde{\mathbf{u}}^{n+1}-\mathbf{u}_{\star \star}^{n}}{\delta t}+\left(\mathbf{u}^{n} \cdot \nabla\right) \tilde{\mathbf{u}}^{n+1}-\eta \nabla \tilde{\mathbf{u}}^{n+1}+\nabla p^{n}=0 .
$$

Taking the inner-product of (4.31) with $2 \delta t \tilde{\mathbf{u}}^{n+1}$, we obtain,

$$
\left\|\tilde{\mathbf{u}}^{n+1}\right\|^{2}-\left\|\mathbf{u}_{\star \star}^{n}\right\|^{2}+\left\|\tilde{\mathbf{u}}^{n+1}-\mathbf{u}_{\star \star}^{n}\right\|^{2}+2 \eta \delta\left\|\nabla \tilde{\mathbf{u}}^{n+1}\right\|^{2}+2 \delta t\left(\nabla p^{n}, \tilde{\mathbf{u}}^{n+1}\right)=0 .
$$

To deal with the pressure term, we take the inner product of (5.8) with $2 \delta t^{2} \nabla p^{n}$ to arrive at

$$
\delta t^{2}\left(\left\|\nabla p^{n+1}\right\|^{2}-\left\|\nabla p^{n}\right\|^{2}-\left\|\nabla p^{n+1}-\nabla p^{n}\right\|^{2}\right)=2 \delta t\left(\tilde{\mathbf{u}}^{n+1}, \nabla p^{n}\right) .
$$


Taking the inner product of (5.8) with $\mathbf{u}^{n+1}$, we obtain

$$
\left\|\mathbf{u}^{n+1}\right\|^{2}+\left\|\mathbf{u}^{n+1}-\tilde{\mathbf{u}}^{n+1}\right\|^{2}=\left\|\tilde{\mathbf{u}}^{n+1}\right\|^{2} .
$$

It follows from (5.8) directly that

$$
\delta t^{2}\left\|\nabla p^{n+1}-\nabla p^{n}\right\|^{2}=\left\|\tilde{\mathbf{u}}^{n+1}-\mathbf{u}^{n+1}\right\|^{2} .
$$

Combining (4.32)-(4.35), we obtain

$$
\left\|\mathbf{u}^{n+1}\right\|^{2}-\left\|\mathbf{u}_{\star \star}^{n}\right\|^{2}+\left\|\tilde{\mathbf{u}}^{n+1}-\mathbf{u}_{\star \star}^{n}\right\|^{2}+\delta t^{2}\left(\left\|\nabla p^{n+1}\right\|^{2}-\left\|\nabla p^{n}\right\|^{2}\right)+2 \eta \delta t\left\|\nabla \tilde{\mathbf{u}}^{n+1}\right\|^{2}=0 .
$$

If we take the inner product of (3.2) with $2 \delta t \mathbf{h}^{n+1}$, we get

$$
2 \delta t M_{1}\left\|\mathbf{h}^{n+1}\right\|^{2}-2 \delta t\left(\mathbf{h}^{n+1},\left(\mathbf{u}_{\star}^{n} \cdot \nabla\right) \mathbf{d}^{n}\right)-2\left(\mathbf{d}^{n+1}-\mathbf{d}^{n}, \mathbf{h}^{n+1}\right)+2 \delta t\left(\mathbf{h}^{n+1},\left(\mathbf{W}_{\star}^{n}+a \mathbf{D}_{\star}^{n}\right) \cdot \mathbf{d}^{n}\right)=0 .
$$

Taking the inner product of (5.4) with $2 \mathbf{u}_{\star}^{n}$, we obtain

$$
\left.\left\|\mathbf{u}_{\star}^{n}\right\|^{2}-\left\|\mathbf{u}^{n}\right\|^{2}+\left\|\mathbf{u}_{\star}^{n}-\mathbf{u}^{n}\right\|^{2}-2 \delta t\left(\mathbf{h}^{n+1} \nabla \mathbf{d}^{n}, \mathbf{u}_{\star}^{n}\right)\right)-\delta t\left(\nabla \cdot\left(\frac{1-a}{2} \mathbf{d}^{n} \mathbf{h}^{n+1}-\frac{1+a}{2} \mathbf{h}^{n+1} \mathbf{d}^{n}\right), \mathbf{u}_{\star}^{n}\right)=0 .
$$

Adding (4.37) with (4.38) and noticing the fact that

$$
\left(\nabla \cdot\left(\frac{1-a}{2} \mathbf{d}^{n} \mathbf{h}^{n+1}-\frac{1+a}{2} \mathbf{h}^{n+1} \mathbf{d}^{n}\right), \mathbf{u}_{\star}^{n}\right)=\left(\mathbf{h}^{n+1},\left(\mathbf{W}_{\star}^{n}+a \mathbf{D}_{\star}^{n}\right) \cdot \mathbf{d}^{n}\right),
$$

we arrive at

$$
\left\|\mathbf{u}_{\star}^{n}\right\|^{2}-\left\|\mathbf{u}^{n}\right\|^{2}+\left\|\mathbf{u}_{\star}^{n}-\mathbf{u}^{n}\right\|^{2}+2 \delta t M_{1}\left\|\mathbf{h}^{n+1}\right\|^{2}-2\left(\mathbf{d}^{n+1}-\mathbf{d}^{n}, \mathbf{h}^{n+1}\right)=0 .
$$

If we take the inner product of (5.5) with $2 \delta t \mu^{n+1}$, we obtain

$$
2\left(\phi^{n+1}-\phi^{n}, \mu^{n+1}\right)+2 \delta t\left(\nabla \cdot\left(\phi^{n} \mathbf{u}_{\star \star}^{n}\right), \mu^{n+1}\right)+2 M_{2} \delta t\left\|\nabla \mu^{n+1}\right\|^{2}=0 .
$$

Taking the inner product of (5.6) with $2 \mathbf{u}_{\star \star}^{n}$, we have

$$
\left.\left\|\mathbf{u}_{\star \star}^{n}\right\|^{2}-\left\|\mathbf{u}_{\star}^{n}\right\|^{2}+\left\|\mathbf{u}_{\star \star}^{n}-\mathbf{u}_{\star}^{n}\right\|^{2}+2 \delta t\left(\mathbf{u}_{\star \star}^{n}, \phi^{n} \nabla \mu^{n+1}\right)\right)=0 .
$$

Adding (4.41) with (4.42), we arrive at

$$
\left\|\mathbf{u}_{\star \star}^{n}\right\|^{2}-\left\|\mathbf{u}_{\star}^{n}\right\|^{2}+\left\|\mathbf{u}_{\star \star}^{n}-\mathbf{u}_{\star}^{n}\right\|^{2}+2\left(\phi^{n+1}-\phi^{n}, \mu^{n+1}\right)+2 M_{2} \delta t\left\|\nabla \mu^{n+1}\right\|^{2}=0 .
$$

Finally, adding up the equations (4.36),(4.40), (4.43) and dividing both side by 2, we obtain

$$
\begin{aligned}
& \frac{1}{2}\left(\left\|\mathbf{u}^{n+1}\right\|^{2}-\left\|\mathbf{u}^{n}\right\|^{2}+\left\|\tilde{\mathbf{u}}^{n+1}-\mathbf{u}_{\star \star}^{n}\right\|^{2}\right)+\frac{1}{2} \delta t^{2}\left(\left\|\nabla p^{n+1}\right\|-\left\|\nabla p^{n}\right\|^{2}\right)+\eta \delta t\left\|\nabla \tilde{\mathbf{u}}^{n+1}\right\|^{2} \\
& +\delta t M_{2}\left\|\nabla \mu^{n+1}\right\|^{2}+\delta t M_{1}\left\|\mathbf{h}^{n+1}\right\|^{2}+\left(\phi^{n+1}-\phi^{n}, \mu^{n+1}\right)-\left(\mathbf{d}^{n+1}-\mathbf{d}^{n}, \mathbf{h}^{n+1}\right)=0 .
\end{aligned}
$$

According to Lemma 4.4

$$
\left(\phi^{n+1}-\phi^{n}, \mu^{n+1}\right)-\left(\mathbf{d}^{n+1}-\mathbf{d}^{n}, \mathbf{h}^{n+1}\right) \geq F^{n+1}-F^{n},
$$

hence, we finally obtain

$$
\begin{aligned}
& \frac{1}{2}\left\|\mathbf{u}^{n+1}\right\|^{2}+F^{n+1}+\frac{\delta t^{2}}{2}\left\|\nabla p^{n+1}\right\|^{2}+\delta t\left(\eta\left\|\nabla \tilde{\mathbf{u}}^{n+1}\right\|^{2}+M_{2}\left\|\nabla \mu^{n+1}\right\|^{2}+M_{1}\left\|\mathbf{h}^{n+1}\right\|^{2}\right) \\
& \leq \frac{1}{2}\left\|\mathbf{u}^{n}\right\|^{2}+F^{n}+\frac{\delta t^{2}}{2}\left\|\nabla p^{n}\right\|^{2} .
\end{aligned}
$$




\section{DeCoupled ENERGy STABle SCHEME FOR A REDUCED MODEL}

In the flow regime where $\mathbf{W}$ and $\mathbf{D}$ are small, a reduced model is obtained as follows:

$$
\left\{\begin{array}{l}
\partial_{t} \mathbf{u}+\mathbf{u} \cdot \nabla \mathbf{u}=-\nabla p+\eta \Delta \mathbf{u}-\phi \nabla \mu-\mathbf{h} \nabla \mathbf{d} \\
\nabla \cdot \mathbf{u}=0 \\
\partial_{t} \mathbf{d}+\mathbf{u} \cdot \nabla \mathbf{d}=M_{1} \mathbf{h} \\
\partial_{t} \phi+\nabla \cdot(\mathbf{u} \phi)=M_{2} \Delta \mu
\end{array}\right.
$$

where

$$
\begin{array}{rrr}
\mu & = & \gamma(\Delta \phi-f(\phi))-K \phi W(\mathbf{d})-\left(A_{1}-A_{2}\right) \nabla \cdot((\mathbf{d} \cdot \nabla \phi) \mathbf{d})-A_{2} \nabla \cdot\left(|\mathbf{d}|^{2} \nabla \phi\right), \\
\mathbf{h}= & \nabla \cdot\left(\frac{K \phi^{2}}{2} \nabla \mathbf{d}\right)+\frac{K \phi^{2}}{2} g^{\prime}(\mathbf{d})-\left(A_{1}-A_{2}\right)(\mathbf{d} \cdot \nabla \phi) \nabla \phi-A_{2}|\nabla \phi|^{2} \mathbf{d} .
\end{array}
$$

An energy dissipation law exists for this model. In [28], a slightly different model in which the phase transport equation is the Allen-Cahn instead of the Cahn-Hilliard equation was studied. A first order energy stable scheme was devised and proved. For this model (eq. (5.1)), we devise a decoupled scheme below following our approach alluded to earlier.

\section{The semi-discrete scheme}

Given the initial conditions $\mathbf{d}^{0}, \phi^{0}, \mathbf{u}^{0}$ and $p^{0}=0$, having computed $\mathbf{d}^{n}, \phi^{n}, \mathbf{u}^{n}$ and $p^{n}$ for $n \geq 0$, we compute $\left(\mathbf{d}^{n+1}, \phi^{n+1}, \mathbf{u}^{n+1}, p^{n+1}\right)$ in the following sequence.

(1) Step 1: update $\mathbf{d}^{n+1}$ :

$$
\left\{\begin{aligned}
\dot{\mathbf{d}}^{n+1}= & M_{1} \mathbf{h}^{n+1} \\
\dot{\mathbf{d}}^{n+1}= & \frac{\mathbf{d}^{n+1}-\mathbf{d}^{n}}{\delta t}+\mathbf{u}_{\star}^{n} \cdot \nabla \mathbf{d}^{n}, \\
\mathbf{h}^{n+1}= & -C_{1}^{n}\left(\mathbf{d}^{n+1}-\mathbf{d}^{n}\right)+\nabla \cdot\left(\frac{K}{2}\left(\phi^{n}\right)^{2} \nabla \mathbf{d}^{n+1}\right)-\frac{K}{2}\left(\phi^{n}\right)^{2} g^{\prime}\left(\mathbf{d}^{n}\right) \\
& -\left(A_{1}-A_{2}\right)\left(\mathbf{d}^{n} \cdot \nabla \phi^{n}\right) \nabla \phi^{n}-A_{2}\left|\nabla \phi^{n}\right|^{2} \mathbf{d}^{n+1}, \\
\left.\frac{\partial \mathbf{d}}{\partial \mathbf{n}}\right|_{\partial \Omega}= & 0,
\end{aligned}\right.
$$

with

$$
\mathbf{u}_{\star}^{n}=\mathbf{u}^{n}-\delta t \mathbf{h}^{n+1} \nabla \mathbf{d}^{n} .
$$

(2) Step 2: update $\phi^{n+1}$ :

$$
\left\{\begin{aligned}
\dot{\phi}^{n+1}= & M_{2} \Delta \mu^{n+1}, \\
\mu^{n+1}= & C_{2}^{n}\left(\phi^{n+1}-\phi^{n}\right)+C_{3}^{n}\left(\Delta \phi^{n+1}-\Delta \phi^{n}\right)+\gamma\left(-\Delta \phi^{n+1}+f^{\prime}\left(\phi^{n}\right)\right) \\
& +\phi^{n+1} W\left(\mathbf{d}^{n+1}\right)-\left(A_{1}-A_{2}\right) \nabla \cdot\left(\left(\mathbf{d}^{n} \cdot \nabla \phi^{n}\right) \mathbf{d}^{n+1}\right)-A_{2} \nabla \cdot\left(\left|\mathbf{d}^{n+1}\right|^{2} \nabla \phi^{n+1}\right), \\
\left.\frac{\partial \phi^{n+1}}{\partial \mathbf{n}}\right|_{\partial \Omega}= & 0,\left.\quad \frac{\partial \mu^{n+1}}{\partial \mathbf{n}}\right|_{\partial \Omega}=0,
\end{aligned}\right.
$$


with

$$
\begin{aligned}
& \dot{\phi}^{n+1}=\frac{\phi^{n+1}-\phi^{n}}{\delta t}+\nabla \cdot\left(\mathbf{u}_{\star \star}^{n} \phi^{n}\right), \\
& \mathbf{u}_{\star \star}^{n}=\mathbf{u}_{\star}^{n}-\delta t \phi^{n} \nabla \mu^{n+1}, \\
& W\left(\mathbf{d}^{n+1}\right)=K\left(\frac{1}{2}\left|\nabla \mathbf{d}^{n+1}\right|^{2}+g\left(\mathbf{d}^{n+1}\right)\right) .
\end{aligned}
$$

(3) Step 3: update $\mathbf{u}^{n+1}$ :

$$
\begin{aligned}
& \left\{\begin{array}{l}
\frac{\tilde{\mathbf{u}}^{n+1}-\mathbf{u}^{n}}{\delta t}+\left(\mathbf{u}^{n} \cdot \nabla\right) \tilde{\mathbf{u}}^{n+1}=\eta \Delta \tilde{\mathbf{u}}^{n+1}-\nabla p^{n}-\phi^{n} \nabla \mu^{n+1}-\mathbf{h}^{n+1} \nabla \mathbf{d}^{n}, \\
\left.\tilde{\mathbf{u}}^{n+1}\right|_{\partial \Omega}=0 .
\end{array}\right. \\
& \left\{\begin{aligned}
\frac{\mathbf{u}^{n+1}-\tilde{\mathbf{u}}^{n+1}}{\delta t} & =-\nabla\left(p^{n+1}-p^{n}\right), \\
\nabla \cdot \mathbf{u}^{n+1} & =0,\left.\quad \mathbf{u}^{n+1}\right|_{\partial \Omega}=0 .
\end{aligned}\right.
\end{aligned}
$$

It can be proved analogously that the scheme is energy stable with respect to any $\delta t$. We omit the details since the proof is identical to the one we discussed above. We comment on this reduced model because it has been a subject of intensive investigations by many numerical analysts and PDE theorists. Physically it is limited, but, it may be of mathematical and numerical values. In the next section, we will focus on the full model, its discretization and a couple of numerical results computed using the model.

\section{Numerical Implementation, Mesh Refinement Results \& Numerical Examples}

The first step (eq. (3.2)) in this decouple scheme is implicit. It involves a variable coefficient fourth order spatial operator with the coefficient proportional to $\delta t$. In order to solve this fourth order equation system, an additional boundary condition must be supplied as alluded to earlier. The solvability condition for this system is not yet established, not mention the uniqueness. However, if we drop the term proportional to $\delta t$ in $\mathbf{u}_{*}^{n}$ and use $\mathbf{u}_{*}^{n}$ instead, the scheme remains first order in time and becomes a fully decoupled elliptic equation system. In the following, we will implement this simplified version of the scheme and study its behavior in mesh refinement.

For the spatial operators in the scheme, we use second-order central finite difference methods to discretize them. The fully discretized equations in the scheme are implemented on GPUs in 3 dimensional space for high-performance computing. For each time-step, we use a precondioned BICG to solve the linear equations, where the preconditioner is solved using FFT. For simplicity in the implementation, we approximate $\mathbf{u}_{\star \star}^{n}$ by $\mathbf{u}^{n}$ as well, which doesn't affect the order-of-accuracy of this scheme, i.e., it is still first-order accurate in time. Numerical tests show that energy decreases in time; so, the discrete energy dissipative law still holds numerically.

We denote the computational domain in space by $[0, L x] \times[0, L y] \times[0, L z]$ where $L x, L y, L z$ are the length in $\mathrm{x}, \mathrm{y}, \mathrm{z}$ directions, respectively. In all the numerical studies presented below, we set

$$
\delta t=2 \times 10^{-4}, \quad \varepsilon=0.01, \quad \delta=0.03, \quad a=1.2, \quad \gamma=10 .
$$

Then, we adopt different values of the other parameters in varying studies. We note a detailed parameter study is essential for investigating the physical properties of this LC model. However, in this paper, we just want to illustrate the effectiveness of our proposed scheme. Therefore, it's not our focus to conduct such a detailed investigation. The choice of $a=1.2$ indicate that the liquid crystal is rodlike. 
6.1. Mesh refinement test in time. In order to have high resolution of spatial mesh size to eliminate the spatial error, here we test the code in $2 \mathrm{D}$ with the spatial mesh-size $512 \times 512$ and time step $\delta t=2 \times 10^{-3}, 10^{-3}, 5 \times 10^{-4}, 2.5 \times 10^{-4}$ and $1.25 \times 10^{-4}$, respectively. At $t=1$, the numerical solutions are compared. Here we calculate the error by treating the numerical result with the finest time step $\left(\delta t=1.25 \times 10^{-4}\right)$ as the accurate solution. The errors in $L_{1}, L_{2}$ and $L_{\infty}$ norms are shown. From the numerical tests, the numerical scheme is shown at least first-order accurate. The results are tabulated in the following tables.

6.2. Example 1: Breakup of liquid crystal filaments. To demonstrate the power of this numerical code, we conduct a numerical simulation of the LC filament breakup phenomenon induced by capillary instability. The computational domain is chosen as $[0,0.25] \times[0,1] \times[0,0.25]$ with grids $128 \times 512 \times 128$. The initial internal orientation of the LCs is parallel to the filament. We denote

$$
r=\sqrt{(x-L x / 2)^{2}+(z-L z / 2)^{2}}, \quad R=0.03+0.005 \sin (2(y-L y / 2)+0.5 \pi) .
$$

The initial profile for the phase function $\phi$ is given by

$$
\phi=\frac{1}{2}\left(\tanh \left(\frac{R-r}{\varepsilon}\right)+1\right) .
$$

The simulation result is shown in Figure 6.1. In this computation, we do not impose any anchoring condition assuming the anchoring effect is weak. The filament eventually breaks up into satellite drops, where each drop shows a pair of defects located at the north and sole pole, respectively. The bead on a string morphology is demonstrated, where we observe multiple small beads near a bigger one connected via thin liquid bridges. In order to observe the $\mathrm{LC}$ orientation and give a better view of interfacial dynamics, LC orientation is shown in $6.1(\mathrm{~h}-\mathrm{n})$, where $2 \mathrm{D}$ slices at $x=0.5 L x$ are plotted. We observe that the LC aligns roughly in the direction of the axis of filament symmetry in the bulk; whereas at the LC-viscous fluid interface, the LC is orthogonal to the normal of the interface at the interface leading to the pair of defects at the north and south pole. This phenomenon agrees qualitatively with the experimental findings.

6.3. Example 2: A sheared liquid crystal drop. In this example, we simulate the sheared rupture of a LC drop. Initially, a spherical LC drop is placed in a viscous fluid matrix with the same density and viscosity as the surrounding viscous fluid matrix. The parallel plates $(y=0, L y)$ moves in the opposite direction with relative speed $v_{0}=12$. One numerical result is shown in Figure 6.2. The drop first elongates due to the shearing flow. After it elongates into a filament, it ruptures into droplets or beads shown in Figure 6.2. We also show a comparison of the rupture phenomenon of LC drops with varying Frank-elastic constant $K$ in Figure 6.4, where we observe that a high Frank-elastic constant facilitates the rupture of the LC drop into more droplets than it is small.

\section{Conclusion}

In this paper, we present a hydrodynamic phase field model derived using the generalized Onsager principle which obeys an energy dissipative law even with the anchoring condition incorporated. We then design a linear, first-order, decoupled, energy stable scheme for the full model and show that the semi-discrete scheme satisfies an analogous, discrete energy dissipation law. While we have only considered the semi-discrete scheme in time, the results can be carried over to fully discrete scheme for any consistent finite element or spectral Galerkin approximations since the proof is based on the variational formulation with all test functions in the same space as the trial function. We further 
implement the scheme with a finite difference method in space on GPUs for high performance computing. Two 3D numerical examples are shown to illustrate the power of this code in resolving complex interfacial fluid dynamics. More detailed studies on dynamics of nematic liquid crystal drops will be given in a sequel.

Acknowledgements. Jia Zhao and Qi Wang are partially supported by NSF-DMS-1200487, NIH2R01GM078994-05A1, AFOSR-FA9550-12-1-0178, and an SC EPSCOR/IDEA award. J. Shen is partially supported by NSF-DMS-1215066 and AFOSR FA9550-11-1-0328. X. Yang is partially supported by NSF-DMS-1200487, AFOSR-FA9550-12-1-0178. In addition, Jia Zhao is also supported by a Dissertation Fellowship from the Provost Office of USC.

\section{REFERENCES}

[1] D. M. Anderson, G. B. McFadden, and A. A. Wheeler. Diffuse-interface methods in fluid mechanics. 30:139-165, 1998.

[2] Franck Boyer and Sebastian Minjeaud. Numerical schemes for a three component Cahn-Hilliard model. ESAIM Math. Model. Numer. Anal., 45(4):697-738, 2011.

[3] J. W. Cahn and J. E. Hilliard. Free energy of a nonuniform system. I. interfacial free energy. J. Chem. Phys., 28:258-267, 1958.

[4] L. Q. Chen and Y. Wang. The continuum field approach to modeling microstructural evolution. JOM, 48:13-18, 1996

[5] N. Condette, C. Melcher, and E. Süli. Spectral approximation of pattern-forming nonlinear evolution equations with double-well potentials of quadratic growth. to appear in Math. Comp.

[6] de Gennes P.-G. and J. Prost. The Physics of Liquid Crystals. Oxford University Press, New York, 1993.

[7] Q. Du, M. Li, and C. Liu. Analysis of a phase field navier-stokes vesicle-fluid interaction model. Dis. Conti. Dyn. Sys.-B, 8(3):539-556, 2007.

[8] J. L. Ericksen. Conservation laws for liquid crystals. Trans. Soc. Rheol., 5:23-34, 1961.

[9] A. Fick. Poggendorff's annalen. Journal of the american mathematics society, pages 59-86, 1855.

[10] J. L. Guermond, P. Minev, and J. Shen. An overview of projection methods for incompressible flows. Comput. Methods Appl. Mech. Engrg., 195:6011-6045, 2006.

[11] M. E. Gurtin, D. Polignone, and J. Viñals. Two-phase binary fluids and immiscible fluids described by an order parameter. Math. Models Methods Appl. Sci., 6(6):815-831, 1996.

[12] D. Jacqmin. Diffuse interface model for incompressible two-phase flows with large density ratios. J. Comput. Phys., 155(1):96-127, 2007.

[13] B. Jerome. Surface effects and anchoring in liquid crystals. Rep. Prog. Phys., 54:391, 1991.

[14] Daniel Kessler, Ricardo H. Nochetto, and Alfred Schmidt. A posteriori error control for the Allen-Cahn problem: circumventing Gronwall's inequality. M2AN Math. Model. Numer. Anal., 38(1):129-142, 2004.

[15] Junseok Kim. Phase-field models for multi-component fluid flows. Commun. Comput. Phys., 12(3):613-661, 2012.

[16] F. M. Leslie. Some constitutive equations for anisotropic fluids. Q. Jl. Mech. Appl. Math., 19:357-370, 1966.

[17] F. M. Leslie. Some constitutive equations for liquid crystals. Arch. Rational Mech. Anal., 28:265-283, 1968.

[18] F. H. Lin. On nematic liquid crystals with variable degree of orientation. Communications on Pure and Applied Mathematics, 44:453-468, 1991.

[19] F. H. Lin. Mathematics theory of liquid crystals, in applied mathematics at the turn of century: Lecture notes of the 1993 summer school, universidat complutense de madrid. 1995.

[20] C. Liu and N.J. Walkington. An Eulerian description of fluids containing visco-hyperelastic particles. Arch. Rat. Mech. Anal., 159:229-252, 2001.

[21] Chun Liu and Jie Shen. A phase field model for the mixture of two incompressible fluids and its approximation by a Fourier-spectral method. Physica D, 179(3-4):211-228, 2003.

[22] John S. Lowengrub and L. Truskinovsky. Quasi incompressible cahn-hilliard fluids and topological transitions. Proceedings of the Royal Society A, 454:2617-2654, 1998.

[23] L. Rayleigh. On the theory of surface forces-II. compressible fluids. Phil. Mag., 33, 1892.

[24] J. Shen and X. Yang. Energy stable schemes for cahn-hilliard phase-field model of two-phase incompressible flows. Chinese Ann. Math. series B, 31:743-758, 2010. 
[25] J. Shen and X. Yang. Numerical approximations of allen-cahn and cahn-hilliard equations. DCDS, Series A, 28:1169-1691, 2010.

[26] J. Shen and X. Yang. A phase-field model and its numerical approximation for two-phase incompressible flows with different densities and viscositites. SIAM J. Sci. Comput., 32:1159-1179, 2010.

[27] Jie Shen. Modeling and numerical approximation of two-phase incompressible flows by a phase-field approach. In Multiscale Modeling and Analysis for Materials Simulation, Lecture Note Series, Vol. 9. IMS, National University of Singapore, 2011, Edited by W. Bao and Q. Du, pages 147-196.

[28] Jie Shen and Xiaofeng Yang. Decoupled energy stable schems for phase field models of two phase complex fluids. SIAM Journal of Scientific Computing, 36(1):122-145, 2014.

[29] J. van der Waals. The thermodynamic theory of capillarity under the hypothesis of a continuous density variation. J. Stat. Phys., 20:197-244, 1893.

[30] X. Yang, J. J. Feng, C. Liu, and J. Shen. Numerical simulations of jet pinching-off and drop formation using an energetic variational phase-field method. J. Comput. Phys., 218(1):417-428, 2006.

[31] X. Yang, M. G. Forest, H. Li, C. Liu, J. Shen, Q. Wang, and F. Chen. Modeling and simulations of drop pinch-off from liquid crystal filaments and the leaky liquid crystal faucet immersed in viscous fluids. J. Comput. Phys., 236:1-14, 2013.

[32] Xiaogang Yang, M. Gregory Forest, and Qi Wang. Near equilibrium dynamics and one-dimensional spatialtemporal structures of polar active liquid crystals. Chinese Physics B, 23(11), 2014.

[33] Xiaogang Yang and Qi Wang. Capillary instability of axisymmetric, active liquid crystal jets. Soft Matter, 10(35):6758-6776, 2014.

[34] P. Yue, J. J. Feng, C. Liu, and J. Shen. A diffuse-interface method for simulating two-phase flows of complex fluids. J. Fluid Mech, 515:293-317, 2004.

[35] Tianyu Zhang, Nick G. Cogan, and Qi Wang. Phase-field models for biofilms i. theory and simulations. SIAM Journal of Applied Mathematics, 69:641-669, 2008.

[36] Jingzhi Zhu, Longqing Chen, Jie Shen, and Veena Tikare. Coarsening kinetics from a variable-mobility cahnhilliard equation: application of a semi-impliciit fourier spectral method. Physical Review E, 60:3564, 1999. 
TABLE 6.1. Error for $\phi$

\begin{tabular}{c|c|c|c|c|c}
\hline$\delta t$ & $2 \times 10^{-3}$ & $1 \times 10^{-3}$ & $5 \times 10^{-4}$ & $2.5 \times 10^{-4}$ & $1.25 \times 10^{-4}$ \\
\hline 1-norm & 1.7238 & 0.8236 & 0.3623 & 0.1235 & - \\
\hline 2-norm & 0.0082 & 0.0039 & 0.0017 & 0.0006 & - \\
\hline$\infty$-norm & $2.228 \times 10^{-4}$ & $1.018 \times 10^{-4}$ & $4.23 \times 10^{-5}$ & $1.35 \times 10^{-5}$ & - \\
\hline
\end{tabular}

TABLE 6.2. Error for $\mathbf{d}_{1}$

\begin{tabular}{c|c|c|c|c|c}
\hline$\delta t$ & $2 \times 10^{-3}$ & $1 \times 10^{-3}$ & $5 \times 10^{-4}$ & $2.5 \times 10^{-4}$ & $1.25 \times 10^{-4}$ \\
\hline 1-norm & 0.9254 & 0.4607 & 0.2069 & 0.0712 & - \\
\hline 2-norm & 0.0059 & 0.0029 & 0.0013 & 0.0004 & - \\
\hline$\infty$-norm & $9.479 \times 10^{-5}$ & $4.273 \times 10^{-5}$ & $1.736 \times 10^{-5}$ & $5.42 \times 10^{-6}$ & - \\
\hline
\end{tabular}

TABLE 6.3. Error for $\mathbf{d}_{2}$

\begin{tabular}{c|c|c|c|c|c}
\hline$\delta t$ & $2 \times 10^{-3}$ & $1 \times 10^{-3}$ & $5 \times 10^{-4}$ & $2.5 \times 10^{-4}$ & $1.25 \times 10^{-4}$ \\
\hline 1-norm & 1.3711 & 0.6019 & 0.2358 & 0.0736 & - \\
\hline 2 -norm & 0.0069 & 0.0030 & 0.0012 & 0.0004 & - \\
\hline$\infty$-norm & $1.35 \times 10^{-4}$ & $6.10 \times 10^{-5}$ & $2.48 \times 10^{-5}$ & $7.6 \times 10^{-6}$ & - \\
\hline
\end{tabular}

TABLE 6.4. Error for $\mathbf{u}_{1}$

\begin{tabular}{c|c|c|c|c|c}
\hline$\delta t$ & $2 \times 10^{-3}$ & $1 \times 10^{-3}$ & $5 \times 10^{-4}$ & $2.5 \times 10^{-4}$ & $1.25 \times 10^{-4}$ \\
\hline 1-norm & 0.2885 & 0.1341 & 0.0574 & 0.0191 & - \\
\hline 2-norm & $5.125 \times 10^{-4}$ & $2.380 \times 10^{-4}$ & $1.016 \times 10^{-4}$ & $3.38 \times 10^{-5}$ & - \\
\hline$\infty$-norm & $4.377 \times 10^{-6}$ & $2.038 \times 10^{-6}$ & $8.70 \times 10^{-7}$ & $2.88 \times 10^{-7}$ & - \\
\hline
\end{tabular}

TABLE 6.5. Error for $\mathbf{u}_{2}$

\begin{tabular}{c|c|c|c|c|c}
\hline$\delta t$ & $2 \times 10^{-3}$ & $1 \times 10^{-3}$ & $5 \times 10^{-4}$ & $2.5 \times 10^{-4}$ & $1.25 \times 10^{-4}$ \\
\hline 1-norm & 0.2651 & 0.1235 & 0.0530 & 0.0177 & - \\
\hline 2-norm & $4.569 \times 10^{-4}$ & $2.126 \times 10^{-4}$ & $9.10 \times 10^{-5}$ & $3.04 \times 10^{-5}$ & - \\
\hline$\infty$-norm & $2.578 \times 10^{-6}$ & $1.194 \times 10^{-6}$ & $5.05 \times 10^{-7}$ & $1.65 \times 10^{-7}$ & - \\
\hline
\end{tabular}




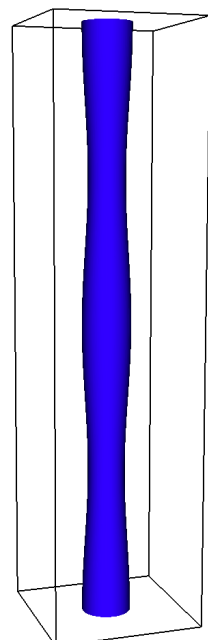

(A)

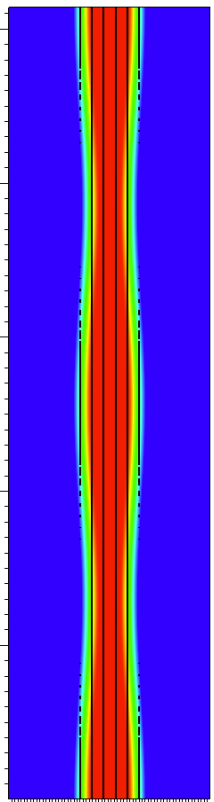

( $\mathrm{H})$

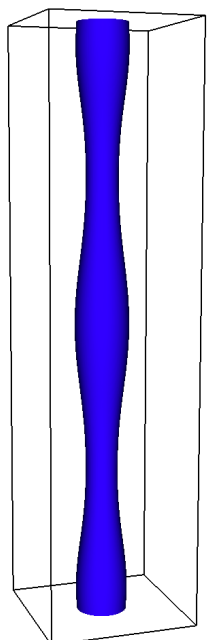

(B)
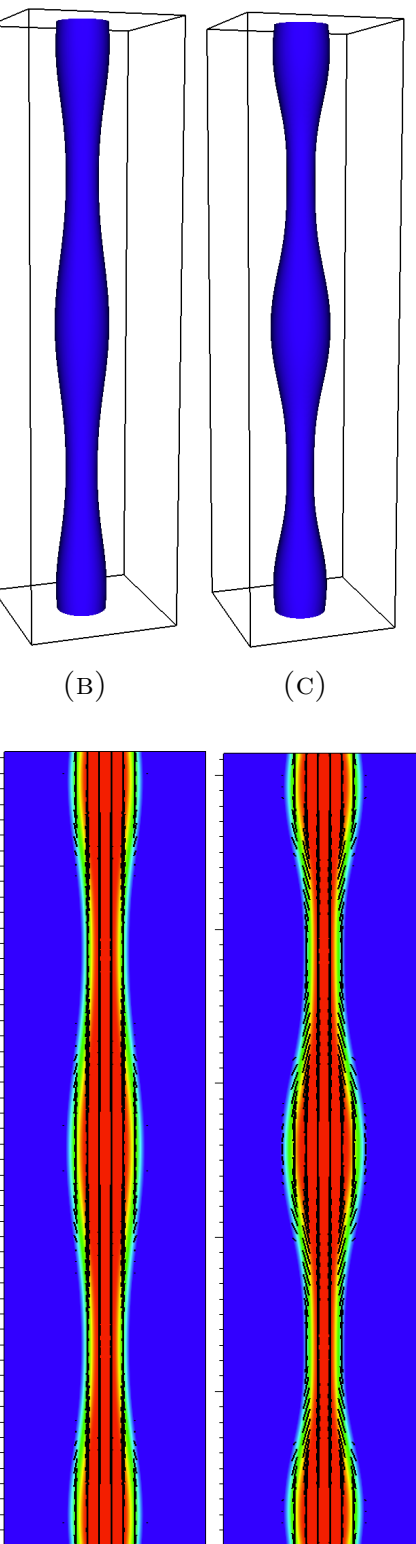

(I)
(C)

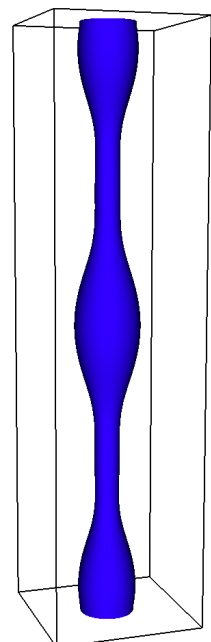

(D)

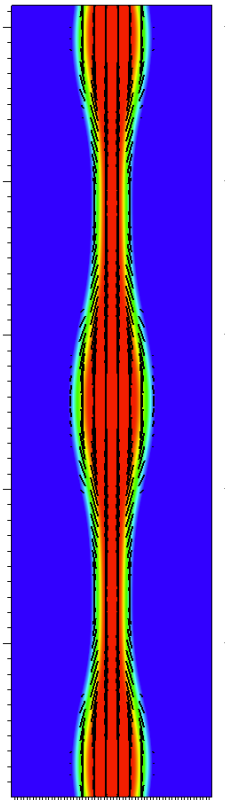

(J)

(K)

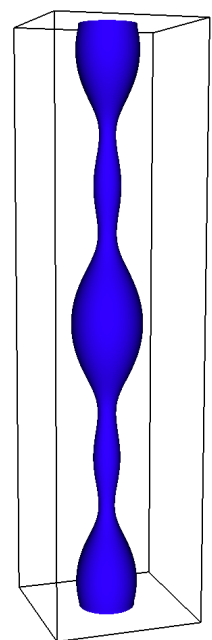

(E)

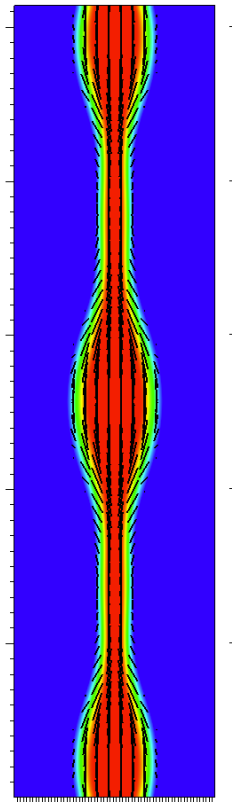

(L)

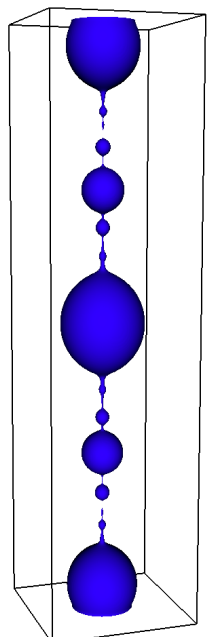

(F)

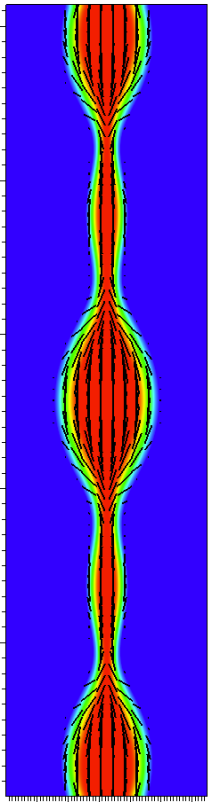

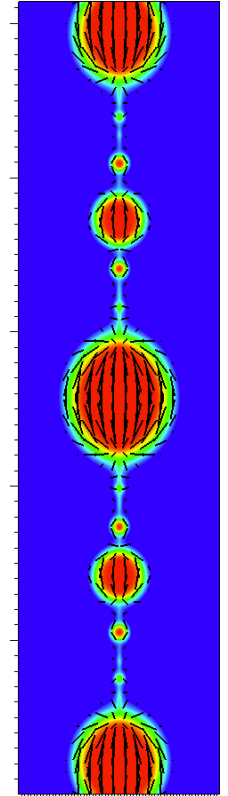

(M)

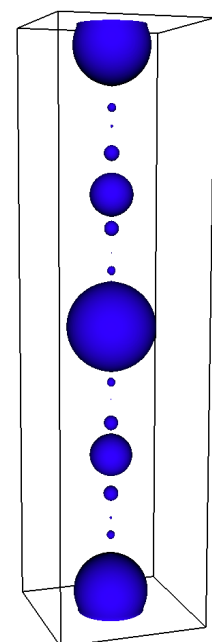

(G)

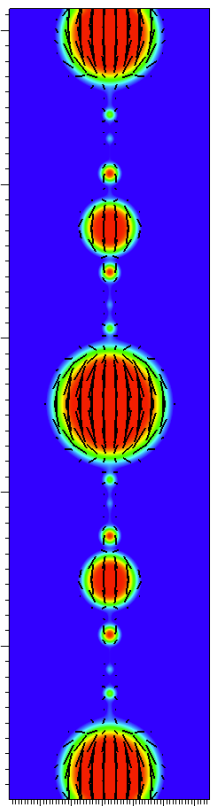

(N)

FiguRE 6.1. Capillary instability of free liquid crystal jets. This figure shows the formation of beads induced by capillary instability with mesh $128 \times 512 \times 128$ and domain size $[0,0.25] \times[0,1] \times[0,0.25]$. A time series $(t=0,1,2,3,4,5,6)$ of the jet in $3 \mathrm{D}$ view is shown in $(\mathrm{A}-\mathrm{G})$ and $2 \mathrm{D}$ view in $(\mathrm{H}-\mathrm{N})$. The parameter values are $\delta t=5.0 \times 10^{-4}, \eta=1.0, M_{1}=1.0 \times 10^{-10}, M_{2}=1.0 \times 10^{-3}, K=0.025$, $A_{1}=A_{2}=0$. 

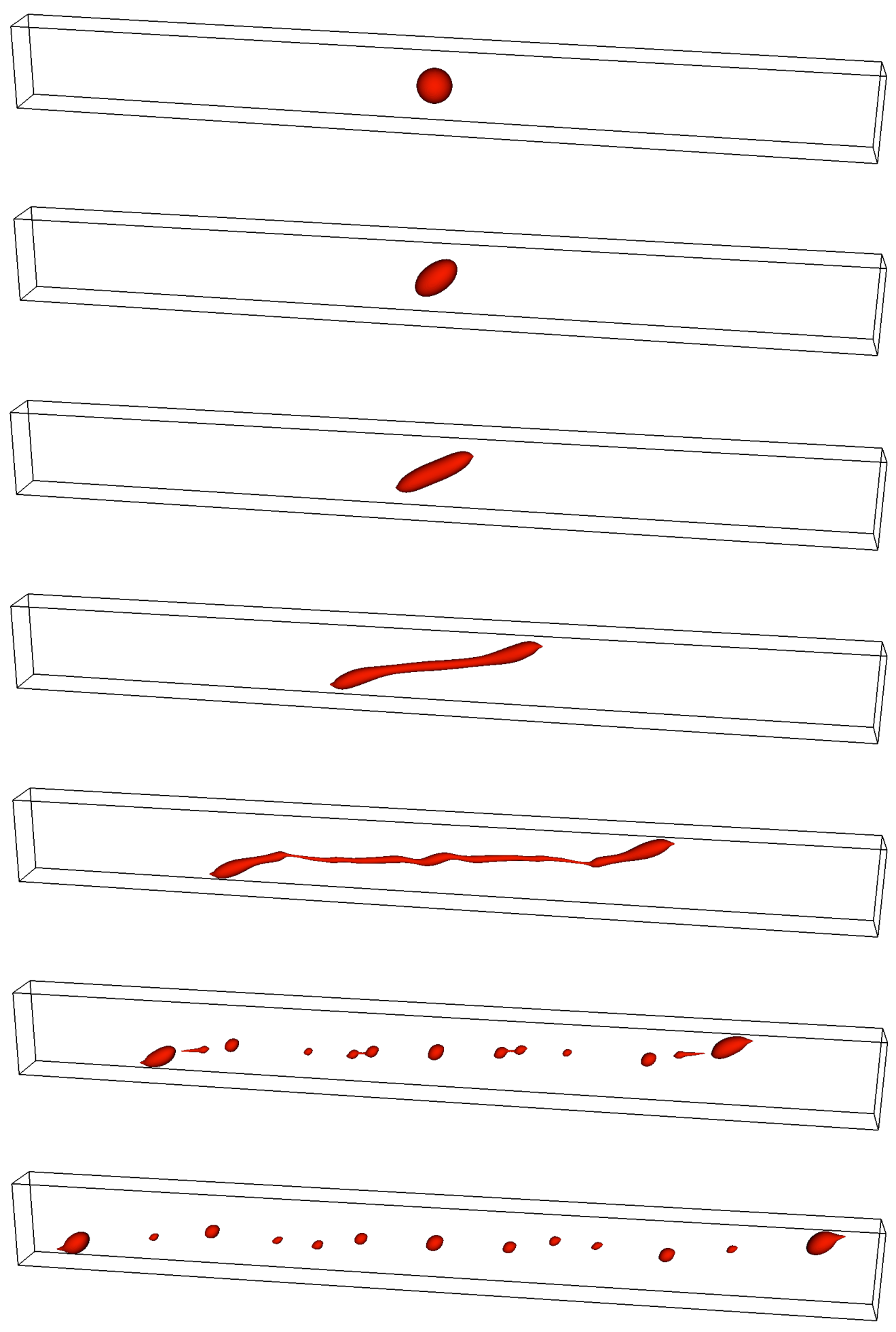

Figure 6.2. 3D simulation of sheared rupture of LC drops immersed in a viscous fluid. This figure shows a time sequence $(t=0,0.4,0.8,1.2,1.6,1.8,2.0)$ of a LC drop immersed in a viscous fluid sheared by a parallel plate flow. The shear rate is 20. The domain is $[0,0.5] \times[0,1] \times[0,10]$ with mesh $64 \times 128 \times 1280, \delta t=5.0 \times 10^{-4}$, $\eta=0.1, M_{1}=2.5 \times 10^{-6}, M_{2}=1.0 \times 10^{-3}, K=0.05, A_{1}=A_{2}=0$. All parameters given are dimensionless. 


\section{○}
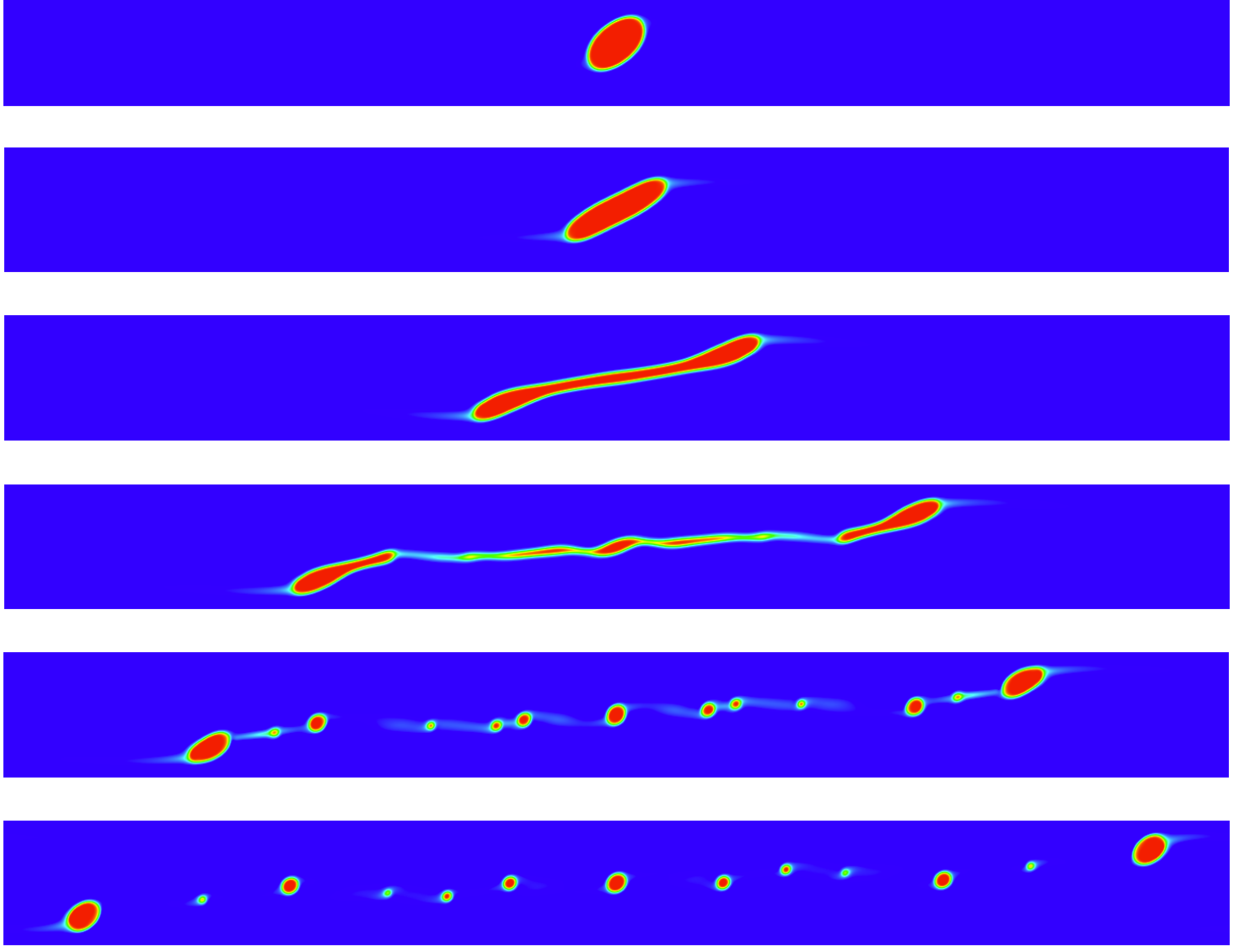

FiguRE 6.3. 3D simulation of sheared rupture of LC drops immersed in a viscous fluid. This shows a $2 \mathrm{D}$ view of the dynamics given in Figure 6.2 at $(t=$ $0,0.4,0.8,1.2,1.6,1.8,2.0)$. 


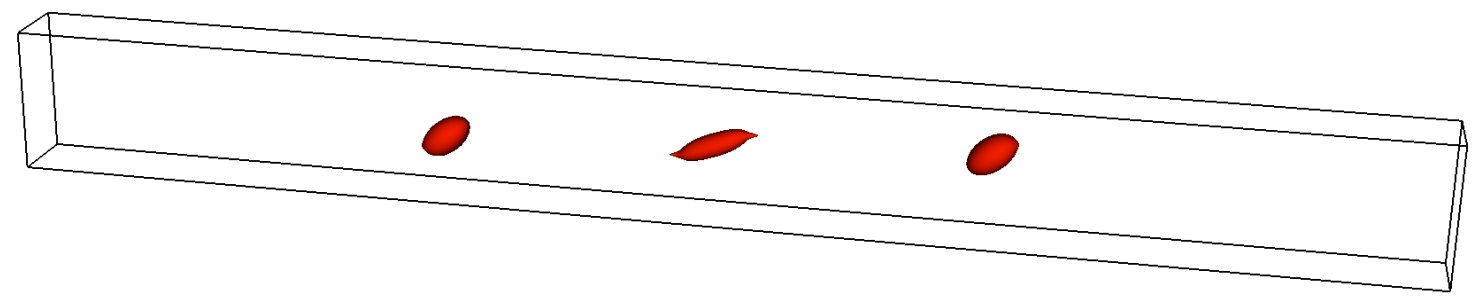

(A) Viscous drop at $t=2.0(K=0)$

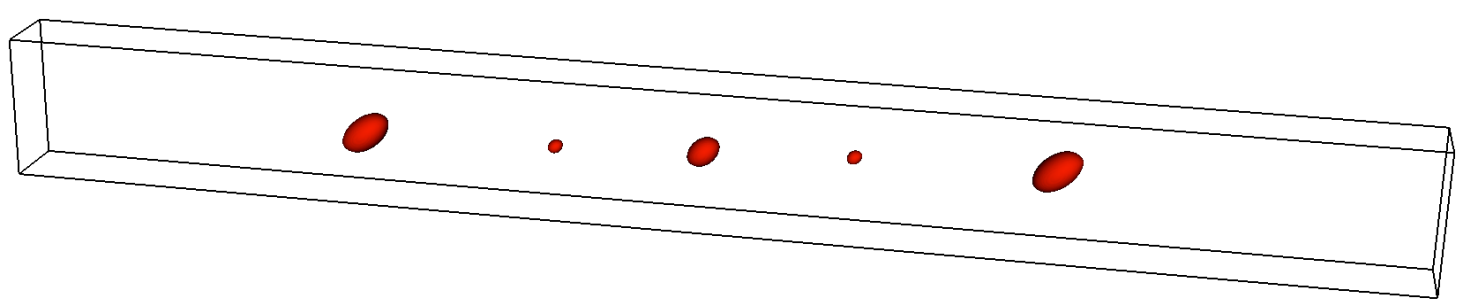

(B) LC drop at $t=2.0(K=0.01)$

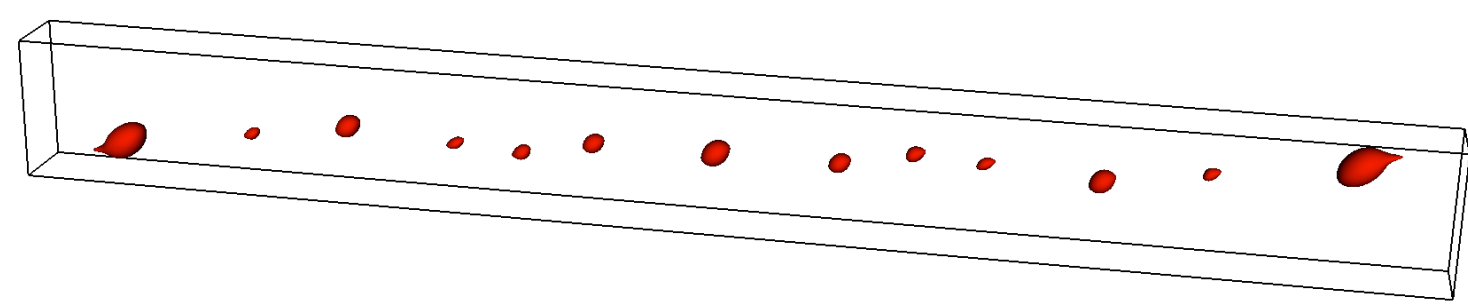

(C) LC drop at $t=2.0(K=0.05)$

Figure 6.4. A comparison of varying $K$ on sheared rupture of LC drops. This figure shows that the distortional elasticity in the LC microstructure tends to create more equal sized LC drops during sheared rupture, a unique feature of the liquid crystal. All the other parameter values are the same as given in Figure 6.2 except:(A) $K=0 ;(\mathrm{B}) K=0.01 ;(\mathrm{C}) K=0.05$. 